\title{
Thermocapillary migration in small-scale temperature gradients: application to optofluidic drop dispensing
}

\author{
Matthieu Robert de Saint Vincent* and Jean-Pierre Delville ${ }^{\dagger}$ \\ Université de Bordeaux, Laboratoire ondes et matière d'Aquitaine (LOMA), \\ UMR CNRS 5798, 351 cours de la Libération, 33405 Talence Cedex, France
}

\begin{abstract}
We experimentally investigate the thermocapillary migration induced by local laser heating of the advancing front of a growing droplet confined in a microfluidic channel. When heating implies an effective increase in interfacial tension, the laser behaves as a "soft door" whose stiffness can be tuned via the optical parameters (beam power and waist). The light-driven thermocapillary velocity of a growing droplet, which opposes to the basic flow, is characterized, for different types of fluid injection, either pressure- or flow-rate-driven, and various channel aspect ratios. Measurements are interpreted using a simplified model for the temperature gradient at the interface, based on a purely diffusive, three-layer system. Considering the mean temperature gradient, we demonstrate that the classical large-scale temperature gradient behavior is retrieved in the opposite case when the thermal gradient length scale is smaller than the droplet size. We also demonstrate that the thermocapillary velocity is proportional to the smallest droplet curvature imposed by the channel confinement. This suggests that the thermocapillary velocity is in fact proportional to the mean temperature gradient and to the largest interface curvature radius, which both coincide with the imposed one and the spherical droplet radius in large-scale and unconfined situations. Furthermore, as used surfactant concentrations are largely above the critical micelle concentration $(\mathrm{CMC})$, we propose a possible explanation, relying on state-of-the-art considerations on highconcentration surfactant-covered interfaces, for the observed effective increase in interfacial tension with temperature. We also propose a mechanism for explaining the blocking effect at the scalinglaw level. This mechanism involves the temporal evolution of hydrodynamic and thermocapillary forces, based on experimental observations. We finally show that this opto-capillary interaction with a microfluidic droplet generator allows for controlling either the flow rate (valve) or the droplet size (sampler), depending on the imposed fluid injection conditions.
\end{abstract}

* Present address: School of Physics and Astronomy, University of St Andrews, North Haugh, Fife, KY16 
9SS, Scotland, UK; Electronic address: mrdsv@st-andrews.ac.uk

$\dagger$ Electronic address: jp.delville@loma.u-bordeaux1.fr 


\section{INTRODUCTION}

Using light to set micro-scale fluid elements in motion is attractive since optical actuation can be totally disconnected to the fluidic system. The very weak amplitude of optical forces [1], typically in the $\mathrm{pN}$ range, nevertheless limits the manipulation capabilities for high-speed microfluidic applications. For instance, optical tweezers [2] have recently been proposed to actuate micropumps composed of optically trapped particles [3, 4], with flow velocities in the $\mu \mathrm{m} \mathrm{s}^{-1}$ range. On the other hand, radiation pressure [5] allows for direct optical manipulation of liquid interfaces, but observation of significant deformations is only possible when interfacial tensions are very small [6] due to the weakness of the photon momentum.

An alternative consists in producing hydrodynamic forces by light rather than using optical forces themselves. Let us consider a bubble, immersed in a viscous light-absorbing fluid. As surface tension depends on temperature, heating locally the interface creates a surface tension gradient that produces a stress (called Marangoni, or thermocapillary) along the interface. This stress induces an interfacial flow, directed toward the region of highest surface tension (generally, the colder), which diffuses in both side of the interface. The motion of the surrounding bulk fluid finally leads to the migration of the bubble in the opposite direction. In a pioneering work, Young et al. [7] gave an expression for the migration velocity (hereafter called "thermocapillary velocity"), and showed that it scales as the bubble radius and the temperature gradient. This expression agrees quantitatively with experiments for bubbles suspended in an infinite medium. These investigations have then been extended to droplets, and the scaling predicted by Young et al. was retrieved in spite of a quantitative discrepancy attributed to the convective transport of heat [8]. The influence of boundaries, considering bubbles squeezed between two parallel plates $[9,10]$, or embedded in long capillaries of various shapes [11-13], were also analyzed. A qualitative difference appears in the case of elongated bubbles, for which the thermocapillary velocity no longer depends on the bubble size.

Later, quantitative discrepancies with Young's results raised the question of impurities influence at interfaces on the thermocapillary migration. Barton and Subramanian [14] observed experimentally that a controlled amount of surfactant inhibits the Marangoni effect. Kim and Subramanian $[15,16]$ theoretically described this inhibition, for insoluble surfactants, in the two extreme cases corresponding to weak and strong interfacial advection. 
The main feature is that surfactant molecules tend to gather onto a stagnant cap, which increases the stiffness of the interface. The inhibiting effect was also observed with soluble surfactants [17]. More recently, Khattari et al. [18] accounted for the dilatational properties of the interface and the diffusive transport of surfactant molecules along the interface. They showed that the presence of surfactant globally leads to (i) a decrease in amplitude of the thermocapillary migration velocity, and (ii) a coupling between the temperature and the interfacial concentration of surfactant molecules. Therefore, the coefficient of interfacial tension variation with temperature, $\partial \sigma / \partial T$, can be written as an "effective" one, accounting for both temperature and concentration effects, including temperature-induced surfactant transport. This effective variation $(\partial \sigma / \partial T)_{\text {eff }}$ can be either positive or negative, depending on the relative strength of the coupling effects.

In the scope of micro-scale fluid manipulation, most studies involve a temperature gradient produced by integrated sources [19-21]. This approach can be seen as the natural scale-reduction of Young's experiment since the temperature gradient remains larger than the typical droplet size. On the other hand, recent works proposed optically-induced thermocapillary approaches to move liquid droplets [22] or even solids [23] floating on a liquid surface. The main advantage is that, as typical radii of laser beams of $1-10 \mu \mathrm{m}$ are simple to implement, producing a large temperature gradient with a weak increase in temperature becomes easy. In a microfluidic environment, we have shown that this method suits well for the realization of an "optical lab on a chip" [24, 25], in which light blocks or deviates [26] the motion of individual water droplets flowing in hexadecane. Applications such as valve, merger, switch, or sorter, have thus been reported. Since the equilibrium interfacial tension is expected to decrease with temperature for a water-hexadecane interface with surfactants [27], droplets should be attracted toward the hot spot. However, experiments [24-27] show a repulsive effect. Verneuil et al. attributed this anomalous behavior to an out-of-equilibrium solutocapillary effect, due to the sudden local depletion of surfactant on the water-oil interface in the vicinity of the laser beam, which opposes and overcomes pure thermocapillarity [27]. The net force resulting from this laser interaction is theoretically [24, 28] and experimentally [27] estimated to be in the range of $0.1 \mu \mathrm{N}$. The laser-induced force equilibrates the force resulting from the upstream fluid, which is experimentally evaluated in an elegant way by introducing a bypass on the relevant part of the microchannel to determine the corresponding hydrodynamic resistance [27]. This estimate, however, does not link the blocking 
force to the main laser input parameters, and therefore cannot quantify the physical effects involved in the blocking of a droplet in a microchannel. On the other hand, in Ref. [24] this force is calculated from the flow field induced, in a depth-averaged Hele-Shaw geometry, by Marangoni stresses, assuming a given analytical temperature distribution.

In fact, contrary to the case of a droplet submitted to an uniform temperature gradient of length scale much larger than the droplet size, where the "effective" temperature gradient experienced by the droplet is obviously the imposed one, in the laser-heating case, the temperature distribution is not uniform, and furthermore its spatial extent is usually smaller than the droplet size. These features raise the question of the temperature gradient seen by the drop to quantitatively study the thermocapillary migration. Moreover, the velocity of a laser-induced thermocapillary migration of a free droplet cannot be directly measured owing to the locality of the laser-fluid interaction. Thus, the purpose of the present investigation is to implement a new way to measure this migration velocity from the competition with the bulk main flow. Indeed, variations in the bulk flow allow for fine scanning of thermocapillary velocities, particularly during the droplet formation.

This paper is organized as follows. The experimental setup is presented in Section II. The fluid properties, geometry of the microchannel and beam characteristics are also summarized. In Section III, we demonstrate how the laser beam is able to slow down or block the advance of the front of a droplet in formation. We measure the velocity of successive droplet fronts, and use this general method to deduce the thermocapillary migration velocity. For a quantitative understanding of the laser-fluid interaction, we also need to characterize the temperature distribution; it is calculated in Section IV. We develop for this purpose an analytical model, which is based on purely diffusive heat transfer and an idealized 2D geometry. The temperature field at the interface is estimated by considering a virtual interface. We finally demonstrate that the thermocapillary velocity can be related to an averaged temperature gradient, and we discuss the influence of the confining geometry. As an application of the blocking effect, we present in Section $\mathrm{V}$ how the laser beam can influence the characteristics (size, frequency of emission) of the emitted droplets, leading to a caliper- or a valve-like behavior depending on the nature of the initial flow, either flow rate- or pressure-driven. 


\section{EXPERIMENTAL SETUP}

Experiments are performed in polydimethylsiloxane (PDMS) microchannels, molded by the standard soft lithography technique [29] and cast onto microscope glass slides. The channels are cross-shaped, with a rectangular cross-section of variable height $(h \simeq 30$ or $50 \mu \mathrm{m})$ and width $(w \simeq 200$ or $100 \mu \mathrm{m})$. As represented in Fig. 1, a continuous Argon-ion laser (Coherent Innova 300C), operating in the $\mathrm{TEM}_{00}$ Gaussian mode at the wavelength in vacuum $\lambda_{0}=514.5 \mathrm{~nm}$, is focused inside the microchannel by different microscope objectives (Olympus), with magnification ranging from $\times 2.5$ to $\times 20$. This leads to values of the beam waist $\omega_{0}$ comprised between 10.3 and $1.4 \mu \mathrm{m}$. The same objective is also used to image the microchannel on a CMOS fast camera (Lightning RTD 16000). A red-pass filter is added in front of the camera to prevent it from any damage due to the laser irradiance.

Water and hexadecane (hereafter called "oil") are pumped in the microchannel. The injection can be controlled at constant flow rate (pipes represented by solid lines in Fig. 1) using syringe pumps, or at constant pressure, by hanging reservoirs at a given altitude (pipes in dashed lines in Fig. 1). A surfactant, the commonly-used sorbitan monooleate (Span 80), is added to the oil phase to avoid wetting of the water phase on the glass slide [30], at a concentration of $2 \%$ in weight. This concentration, quite classical in microfluidics, is more than 30 times the critical micelle concentration (CMC) [27, 31]. The resulting interfacial tension is $\sigma \simeq 5 \mathrm{mN} \mathrm{m}^{-1}$. As previously reported, for this concentration range the interfacial flow is directed toward the hot spot [24], reflecting a positive value of the "effective" variation of interfacial tension with temperature, $(\partial \sigma / \partial T)_{\text {eff }}$, which leads to a repulsing thermocapillary effect. This point will be detailed below (Section V).

As both fluids are transparent at the laser wavelength, a dye, uranine (disodium fluorescein), is added in water at $0.1 \%$ in weight. This fluorescent dye has a quantum yield of $50 \%$ : half of the absorbed energy is restored as light, allowing for visualizing the spot position, and the other part is dissipated as heat. The resulting optical absorption of the solution at 514.5 $\mathrm{nm}$ was measured to be $\alpha=(1.764 \pm 0.010) \mathrm{mm}^{-1}$. Additionally, several experiments were performed with a commercial Food Red 3 dye solution (E122: azorubine, Vahiné, France). Therefore, water is the only fluid which is directly heated by the laser beam. Furthermore, water has a refractive index $(n=1.33)$ lower than the hexadecane one $(1.43)$, so the laser light is totally reflected in hexadecane by the curved menisci at high values of the incidence 


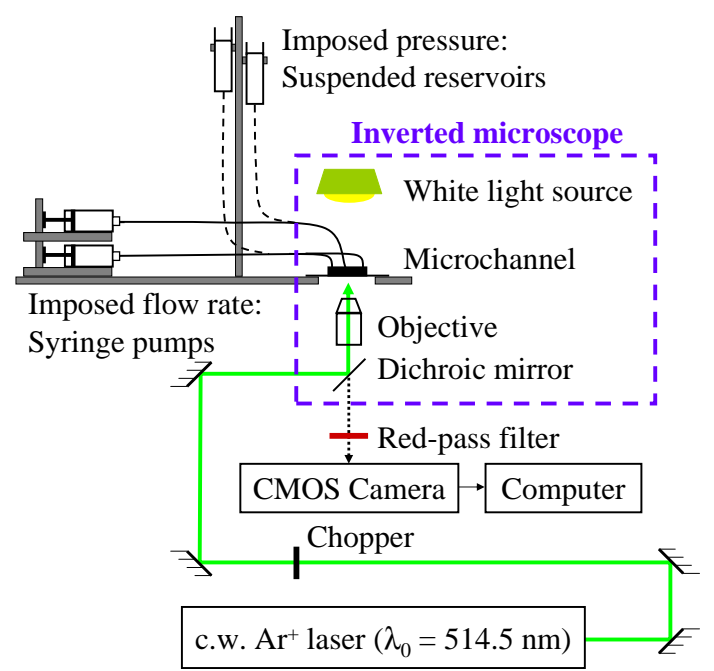

Figure 1. Schematics of the experimental setup.

angle. Finally, the dye and surfactant concentration are sufficiently weak to consider fluids as homogeneous from the point of view of thermodynamics and heat transfer.

\section{THERMOCAPILLARY VELOCITY MEASUREMENTS}

Water drops are emitted in the oil phase by hydrodynamic focusing [32, 33]. Water is injected by the central main channel, and oil by the two lateral ones. Figure 2 (a) shows the droplet formation in the cross region. The advance of the water front, initially slow, accelerates when the front overtakes the intersection. At the same time, at the back of the droplet the interface forms a neck, which thins and eventually breaks up. A drop is thus released, and the same process repeats. For the chosen couple of imposed pressures, the whole process typically lasts $\sim 2 \mathrm{~s}$.

Beam axis is then set up in the centerline of the main channel, downstream from the microchannel cross, and the laser is turned on. As shown in Fig. 2 (b), the laser has no effect on the advance of the interface before it reaches the spot position, hexadecane being optically transparent. Then, if the beam power is high enough, the interface may be blocked during several seconds, and finally released.

The video frames allow us to determine the instantaneous velocity of the front interface of successive droplets. When the interface is submitted to the laser, the measured velocity is the sum of the velocity resulting from the basic flow, $u_{0}$, imposed by the syringe pumps 


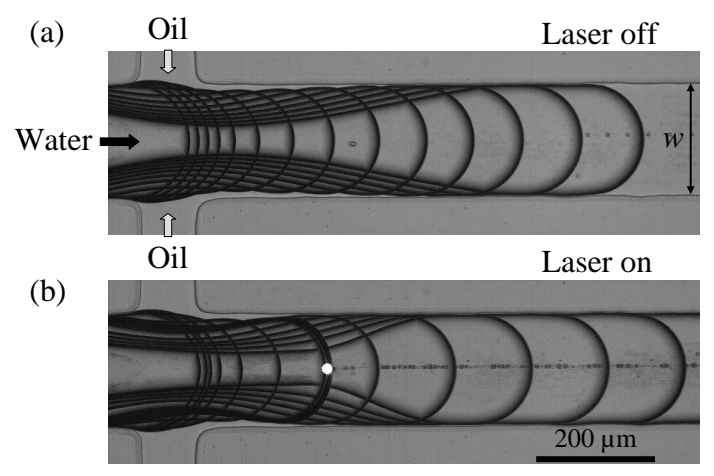

Figure 2. Superposition of successive frames illustrating the advance of a droplet front at constant pressure during the formation process, (a) without and (b) with laser. The beam is located at the white dot. Time delay between the first and the last frames are respectively $1.04 \mathrm{~s}$ (laser off) and $4.72 \mathrm{~s}$ (laser on), with a time increment of $0.08 \mathrm{~s}$ between two successive frames. The channel width is $w \simeq 200 \mu \mathrm{m}$; the imposed pressures are $1835 \mathrm{~Pa}$ (oil) and $1875 \mathrm{~Pa}$ (water).

or the hydrostatic pressure, and the velocity resulting from the laser-droplet interaction (as previously defined, the "thermocapillary velocity", $\left.u_{\mathrm{th}}\right)$. The three following cases should be considered:

i. $u_{\mathrm{th}}<u_{0}$ : The interface is slowed down, and overtakes the laser beam without being blocked;

ii. $u_{\mathrm{th}}=u_{0}$ : Corresponds to the equilibrium;

iii. $u_{\mathrm{th}}>u_{0}$ : The interface should move backward to an equilibrium position, where it turns to case ii. This position is far enough from the beam axis for equating the velocities, and close enough for light being still refracted.

We propose to deduce the thermocapillary velocity from the measurement of the velocity of the front interface at the beam location. The blocking of a first droplet can strongly modify the flow conditions, thus inducing fluctuations in the front velocity of the following droplets in formation. This velocity can range, successively, from below to above the thermocapillary velocity, which is assumed to depend on laser parameters (beam power and waist) only.

We measure the instantaneous velocity of the front interface of successive droplets during the formation process, as plotted by solid lines in Fig. 3. Each solid line represents an individual droplet, the line being broken when the droplet advancing front leaves the field 


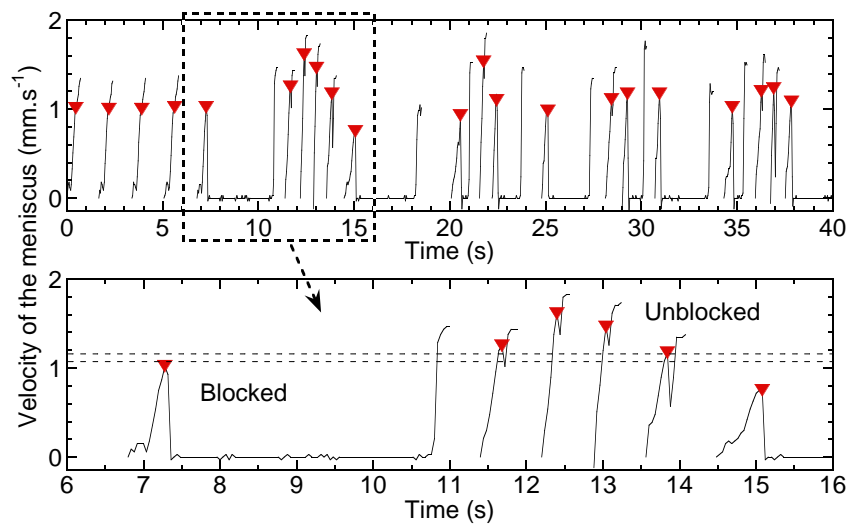

Figure 3. Velocity of 21 successive droplet fronts, for a pressure-driven flow. Each solid line segment represents one individual droplet. The laser is turned on at $t \simeq 6.5 \mathrm{~s}$. Symbols indicate the velocity at the beam position. Top: full acquisition, bottom: close-up corresponding to the $(6-16)$ s interval. Dashed lines correspond to the velocities of the slowest unblocked (top) and the fastest blocked (bottom) drops. Beam power is $P=49 \mathrm{~mW}$, and $\omega_{0}=2.85 \mu \mathrm{m}$.

of view, prior to the detection of the following droplet front. To illustrate the method, let us consider the velocity at the beam location, represented by the symbols. The laser is turned on at $t \simeq 6.5 \mathrm{~s}$. The interface is blocked, the water volume grows, clogs the oil flow, and a drop is eventually released. Due to interface blocking, fluid obstruction, and drop generation, the flows are strongly disturbed and thus produce large velocity fluctuations of the front interface of the following droplet in formation. We note that the blocked droplets are the slowest ones: a maximal blocking velocity can be determined (lower dashed line on the bottom plot of Fig. 3). On the other hand, the fastest droplets may feel the laser but are never blocked: a minimum non-blocking velocity is also reported (upper dashed line on the same plot). From this velocity range, we can confidently define a thermocapillary velocity as the mean of these two extreme values. In the case represented in Fig. 3, thermocapillary velocity is thus comprised between 1.07 and $1.16 \mathrm{~mm} \mathrm{~s}^{-1}$, i.e. $1.12 \mathrm{~mm} \mathrm{~s}^{-1}$.

This measurement is repeated for a wide range in beam power (between the blocking threshold and the boiling point of the aqueous solution), and for different beam waists. As illustrated in the inset of Fig. 4, pressure-driven and rate-driven flows lead to very close values of the thermocapillary velocity, which is totally consistent with the fact that thermocapillary velocities are not related to the injection method. The average value between them is thus considered hereafter. The so-determined thermocapillary velocities are represented 


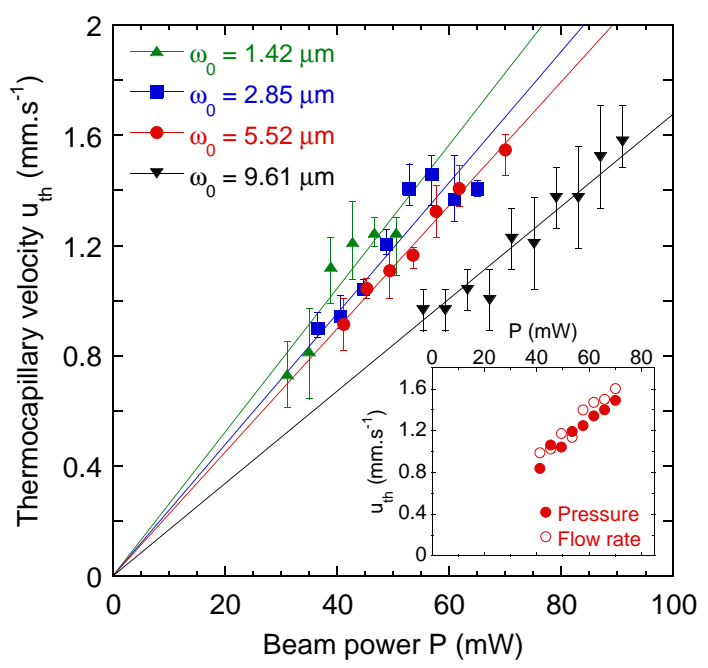

Figure 4. Thermocapillary velocity deduced from the successive droplet front velocity, for 4 different values of the beam waist. Data corresponding to pressure-driven and rate-driven flows are mixed. The channel is $200 \mu \mathrm{m}$ wide and $30 \mu \mathrm{m}$ high. Inset: data corresponding to $\omega_{0}=5.52 \mu \mathrm{m}$, for the pressure-driven (filled symbols) and flow-rate-driven (open symbols) cases.

by symbols in Fig. 4, where the error bars give the interval between the slowest unblocked and the fastest blocked drops. We can see a linear increase of this velocity with beam power, the slope of which increasing weakly when decreasing the beam waist. This behavior should be understood by calculating the distribution of temperature. Note the strength of this method in the sense that flow rate or pressure values are not important as far as some drops may be blocked and others not, in the accessible beam power range.

\section{THERMOCAPILLARY VELOCITY BEHAVIOR}

\section{A. Temperature gradient at the interface}

To interpret the velocity variations presented in Fig. 4 within the framework of thermocapillary actuation, we need to determine the temperature distribution at the interface, and thus calculate the laser-heating of a thin liquid layer sandwiched between two solids (Fig. 5). We deduce the values of the temperature field, and the temperature gradient, on a virtual meniscus located at the place of the real one. The so-calculated temperature gradient is finally averaged.

A side view of the droplet front is represented in Fig. 5 (a). The beam crosses successively 
(a)

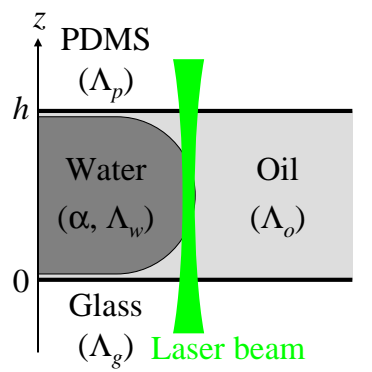

(b)

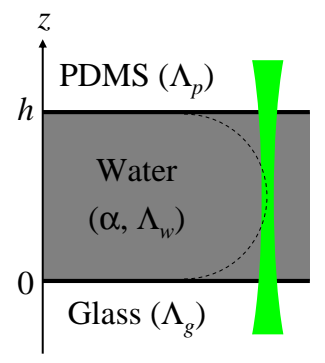

Figure 5. (a) Side view of the laser-meniscus interaction. Glass and PDMS layers are assumed transparent and semi-infinite; optical absorption only occurs in water $\left(\alpha=1.764 \mathrm{~mm}^{-1}\right.$ with uranine). Thermal conductivities are, in $\mathrm{W} \mathrm{K}^{-1} \mathrm{~m}^{-1}, 1.38$ (glass), 0.6 (water), 0.14 (oil, based on the value of pure hexadecane), and 0.15 (PDMS). (b) Idealization of the geometry. The dashed line represents the virtual meniscus (see text).

a glass slide, an ultra-thin layer of oil, a thin layer of water (absorbing part of the energy), another ultra-thin layer of oil, and finally a thick layer of PDMS - thick glass and PDMS layers are considered as semi-infinite. In order to keep the cylindrical symmetry of the heating beam, the section is idealized as represented in Fig. 5 (b). The quasi-matching in thermal conductivities between oil and PDMS allow us to assimilate the oil phase and the side walls of the microchannel. Furthermore, the lubrication oil film between water and glass, which should reduce the heat transfer, is not taken into account, owing to its small thickness. This approximation is discussed in the Appendix A. Finally, our idealized situation neglects the presence of the water-oil interface by considering a virtual meniscus in a pure water layer. As illustrated in the Appendix B, this approximation leads to a $10 \%$ overestimation of the calculated laser overheating compared to the experimental situation.

Since (i) the width of the channel is larger than its height, and (ii) thermal boundary conditions apply at the bottom glass slide and the top PDMS roof, the height will define the characteristic thermal length scale and the lateral walls can be ignored.

From an optical point of view, the Rayleigh length $\lambda_{R}=n \pi \omega_{0}^{2} / \lambda_{0}$ is larger than the channel height, so the beam can be considered as cylindrical along its propagation through the channel [34]. The calculation is thus made in the cylindrical system of coordinates attached to the laser beam, with a symmetry of revolution as stated above. Moreover, the absorption of the water layer is weak, $\alpha h \ll 1$, so the vertical dependance of light intensity 
over the channel height can be neglected. The laser intensity profile can thus be written as $I(r)=2 P /\left(\pi \omega_{0}^{2}\right) \mathcal{G}(r)$, where $\mathcal{G}(r)=\mathrm{e}^{-2 r^{2} / \omega_{0}^{2}}$ is the Gaussian enveloppe.

The thermal length scale, $\ell_{T} \sim h / 2$, and the typical fluid velocities in the $\mathrm{mm} \mathrm{s}^{-1}$ range, lead to a thermal Péclet number $\mathrm{Pe} \sim u \ell / D_{T}$ of the order of 0.1 (the thermal diffusivity of water being $D_{T}=1.4 \times 10^{-7} \mathrm{~m}^{2} \mathrm{~s}^{-1}$ ), allowing to neglect the convective transport of heat. Furthermore, the characteristic heating time $\ell_{T}^{2} / D_{T}$ is in the millisecond range, which is very small compared to the time scales relevant in our experiments ( 0.1 to several seconds). We can thus consider steady-state heat diffusion equations in the three-layer system (subscripts $w, g$ and $p$ denote, respectively, water, glass, and PDMS),

$$
\begin{aligned}
& \left(\nabla_{r}^{2}+\frac{\partial^{2}}{\partial z^{2}}\right) T_{g}=0 \\
& \left(\nabla_{r}^{2}+\frac{\partial^{2}}{\partial z^{2}}\right) T_{w}+\frac{2 P}{\pi \omega_{0}^{2}} \frac{\alpha}{\Lambda_{w}} \mathcal{G}(r)=0 \\
& \left(\nabla_{r}^{2}+\frac{\partial^{2}}{\partial z^{2}}\right) T_{p}=0
\end{aligned}
$$

Here $\nabla_{r}^{2}=(1 / r)(\partial / \partial r)(r \partial / \partial r)$ is the radial part of the Laplacian operator, and $\Lambda$ the thermal conductivity. Considering the cylindrical symmetry, classical resolution methods use Fourier-Bessel decomposition [35]. In the Fourier-Bessel space, the temperature field is defined as

$$
\tilde{T}(k, z)=\int_{0}^{\infty} T(r, z) J_{0}(k r) r \mathrm{~d} r
$$

with $J_{0}(k r)$ the zero-th order Bessel's function of the first kind. Heat diffusion equations become

$$
\left(\frac{\partial^{2}}{\partial z^{2}}-k^{2}\right) \tilde{T}_{w}+\frac{2 P}{\pi \omega_{0}^{2}} \frac{\alpha}{\Lambda_{w}} \tilde{\mathcal{G}}(k)=0
$$

in water, and

$$
\left(\frac{\partial^{2}}{\partial z^{2}}-k^{2}\right) \tilde{T}_{g, p}=0
$$

elsewhere. The variables marked with a tilde are the Fourier-Bessel transforms of the unmarked ones. Especially, we notice that $\tilde{\mathcal{G}}(k)=\left(\omega_{0} / 2\right)^{2} \mathrm{e}^{-k^{2} \omega_{0}^{2} / 8}[36]$.

We impose the following interfacial boundary conditions: continuity of the temperature fields,

$$
\begin{aligned}
& \tilde{T}_{w}(k, z=0)=\tilde{T}_{g}(k, z=0) \\
& \tilde{T}_{w}(k, z=h)=\tilde{T}_{p}(k, z=h),
\end{aligned}
$$


and of the heat fluxes,

$$
\begin{aligned}
& \Lambda_{w}\left(\frac{\partial \tilde{T}_{w}}{\partial z}\right)_{(k, z=0)}=\Lambda_{g}\left(\frac{\partial \tilde{T}_{g}}{\partial z}\right)_{(k, z=0)} \\
& \Lambda_{w}\left(\frac{\partial \tilde{T}_{w}}{\partial z}\right)_{(k, z=h)}=\Lambda_{p}\left(\frac{\partial \tilde{T}_{p}}{\partial z}\right)_{(k, z=h)},
\end{aligned}
$$

across the interfaces.

Solutions of eqs. 5 and 6 are

$$
\begin{aligned}
& \tilde{T}_{g}(k, z)=A_{g}(k) \mathrm{e}^{k z} \\
& \tilde{T}_{w}(k, z)=A_{w}(k) \mathrm{e}^{k z}+B_{w}(k) \mathrm{e}^{-k z}+\frac{\alpha}{\Lambda_{w}} \frac{\tilde{I}(k)}{k^{2}} \\
& \tilde{T}_{p}(k, z)=B_{p}(k) \mathrm{e}^{-k z}
\end{aligned}
$$

with $\tilde{I}(k)=2 P /\left(\pi \omega_{0}^{2}\right) \tilde{\mathcal{G}}(k)$. The constants of integration $A(k)$ and $B(k)$ are determined from the boundary conditions:

$$
\begin{aligned}
& A_{g}(k)=-\frac{\alpha}{\Lambda_{w}} \frac{\tilde{I}(k)}{k^{2}} \mathcal{F}(k) \\
& A_{w}(k)=-\frac{\alpha}{2 \Lambda_{w}} \frac{\tilde{I}(k)}{k^{2}} \mathcal{F}_{+}(k) \\
& B_{w}(k)=-\frac{\alpha}{2 \Lambda_{w}} \frac{\tilde{I}(k)}{k^{2}} \mathcal{F}_{-}(k) \\
& B_{p}(k)=\frac{\alpha}{2 \Lambda_{p}} \frac{\tilde{I}(k)}{k^{2}}\left(\mathcal{F}_{+}(k) \mathrm{e}^{2 k h}-\mathcal{F}_{-}(k)\right),
\end{aligned}
$$

with $\left(\right.$ we set $\left.\Lambda_{i j}=\Lambda_{i} / \Lambda_{j}\right)$

$$
\mathcal{F}(k)=\frac{\left(1-\Lambda_{p w}\right) \mathrm{e}^{-2 k h}+2 \Lambda_{p w} \mathrm{e}^{-k h}-\left(1+\Lambda_{p w}\right)}{\left(1+\Lambda_{p w}\right)\left(1+\Lambda_{g w}\right)-\left(1-\Lambda_{p w}\right)\left(1-\Lambda_{g w}\right) \mathrm{e}^{-2 k h}}
$$

and

$$
\mathcal{F}_{ \pm}(k)=1+\left(1 \pm \Lambda_{g w}\right) \mathcal{F}(k)
$$

We deduce the temperature field in real space by computing the inverse Fourier-Bessel transform. In water, we get

$$
\begin{aligned}
T_{w}(r, z) & =\frac{\alpha P}{2 \pi \Lambda_{w}} \int_{0}^{\infty} \frac{\mathrm{e}^{-k^{2} \omega_{0}^{2} / 8}}{k} \\
& \times\left(1-\frac{\mathcal{F}_{+}(k)}{2} \mathrm{e}^{k z}-\frac{\mathcal{F}_{-}(k)}{2} \mathrm{e}^{-k z}\right) J_{0}(k r) \mathrm{d} k
\end{aligned}
$$




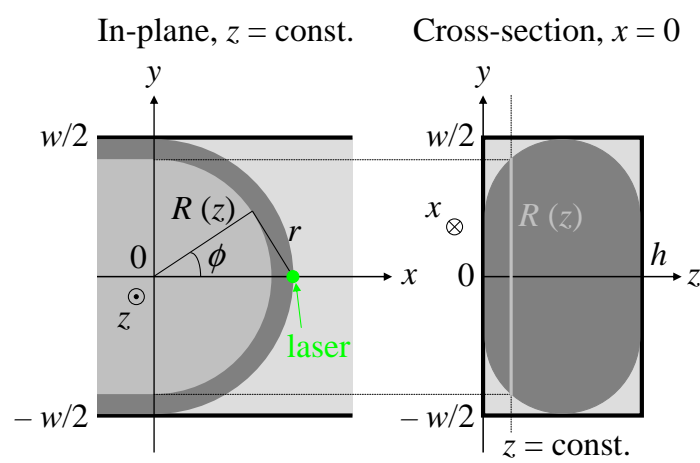

Figure 6. Representation of the virtual meniscus, top $(z=$ const. $)$ and cross-sectional $(x=0)$ views.

This expression is numerically computed with MATLAB ${ }^{\circledR}$, for successive $z=$ const. slices, giving the temperature rise at any radial distance from the beam axis. In a second step we must determine the temperature at each point of a virtual interface located in place of the real one.

The interface can be schematized as represented in Fig. 6. In a given $z=$ const. plane, the front of the droplet draws a half-circle of radius $R(z)$. A polar system of coordinates $(R(z), \phi)$ is attached to its center. Neglecting the lubrication films, the channel geometry leads to

$$
\begin{aligned}
& R(z)=\frac{w-h}{2}+\sqrt{z(h-z)} \\
& \phi=\arccos \left(\frac{w^{2} / 4-r^{2}+R^{2}(z)}{w R(z)}\right) .
\end{aligned}
$$

We can now determine, for each $z=$ const. slice, both the temperature distribution $T_{z}(\phi)$ and the azimuthal temperature gradient $1 / R(z)(\partial T / \partial \phi)_{z}$, along the virtual interface.

The rise in temperature, represented in Fig. 7 (a) for the $z=h / 2$ plane, decreases sharply with the polar angle. A small dependance in beam waist can be noticed at small polar angles. Moreover, the radial distribution of temperature deviates from a purely Gaussian form, imposed by the beam shape, after a distance of about $\omega_{0}$, as illustrated in the right inset of Fig. 7 (a). The largest amplitude of the heating on the beam axis, normalized to the beam power, ranges from 0.85 to $1.72 \mathrm{~K} \mathrm{~mW}^{-1}$ for decreasing beam waists. The boiling point of water should be reached for a temperature rise of about $80 \mathrm{~K}$, i.e. for beam powers between $47 \mathrm{~mW}$ (for $\omega_{0}=1.42 \mu \mathrm{m}$ ) and $95 \mathrm{~mW}$ (for $\omega_{0}=9.61 \mu \mathrm{m}$ ), which are very close to experimental observations (respectively 51 and $91 \mathrm{~mW}$ ). At higher powers, we 


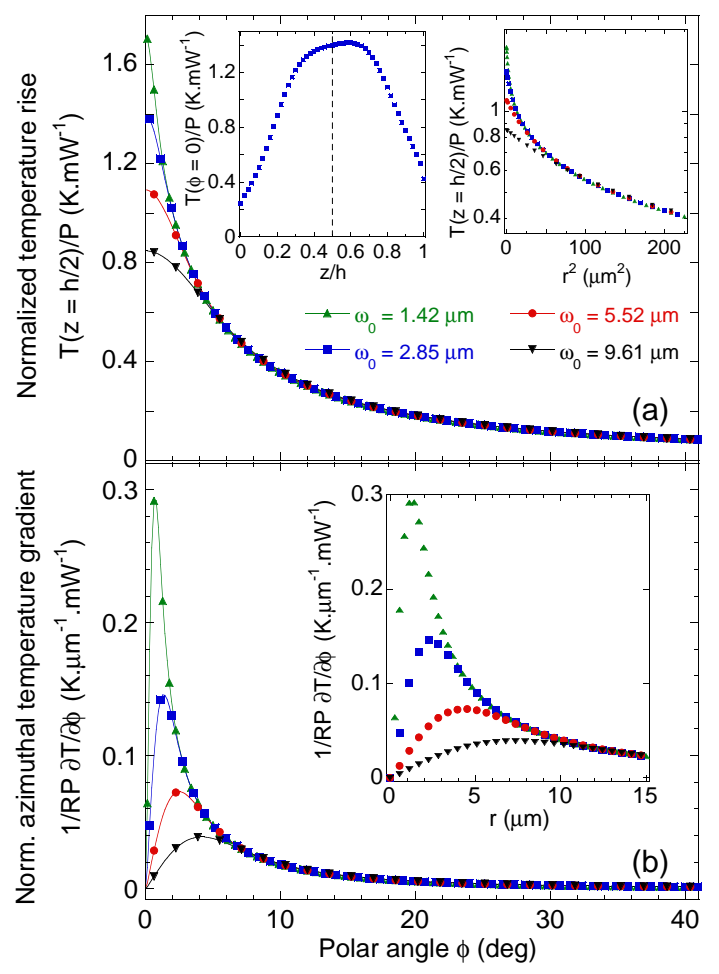

Figure 7. (a) Mid-plane distribution of the laser-induced temperature rise on the meniscus, for the 4 beam waists considered. Data are normalized by the beam power. Left inset shows the vertical evolution of interfacial temperature, at $\phi=0$, for $\omega_{0}=2.85 \mu \mathrm{m}$. Right inset: mid-plane temperature plotted in logarithmic scale against the square of the distance to the beam axis. (b) Mid-plane values of the azimuthal temperature gradient on the meniscus. Data are normalized by the beam power. Inset is a close-up view of the near-axis values plotted against the distance to the beam axis. Geometrical parameters are $w=200 \mu \mathrm{m}, h=30 \mu \mathrm{m}$.

observed the repeated nucleation of bubbles near the beam axis. This observation supports the relevance of the used approximations. Despite its simplicity, this model catches quite well the experimental features. Even if a more refined numerical calculation could have been very accurate, we highlight the fact that an analytical calculation scheme is essential to provide a simple and accurate estimate of the temperature gradient, and predict some expected behaviors.

The left inset of Fig. 7 (a) shows the predicted vertical dependance of the rise in interfacial temperature, in the $(x, z)$ plane. The temperature distribution varies smoothly in the vicinity of the midplane, but decreases sharply near the up (PDMS) and down (glass) boundaries. This vertical dependance mainly results from the variation in radial distance to the beam 
axis. Moreover, the vertical temperature distribution has an asymmetric shape, with higher values in the upper part of the channel. This part is bounded by PDMS, which has a thermal conductivity smaller than glass (bounding the down part of the liquid layer). Thermal losses are thus less important at the upper boundary than at the bottom one.

The distribution of the azimuthal temperature gradient, represented in Fig. 7 (b) for the $z=h / 2$ plane, presents a peak, inversely proportional to the beam waist, at a distance from beam axis of the order of $\omega_{0}$ [see inset of Fig. 7 (b)]. At larger distances, the gradient strongly attenuates, with the same shape whatever the beam waist, and rapidly tends to zero, justifying by the way the fact that lateral channel boundaries can be neglected. Local values as high as several $\mathrm{K}_{\mu \mathrm{m}}^{-1}$ can be reached for the typical involved intensities. These features imply that thermally-induced interfacial flows should be very strong, and localized close to the beam axis.

In a third step we calculate the mean temperature gradient on the meniscus interface. In each plane $z=$ const., the averaging should be performed on a path corresponding to the whole set of points lying to the interface up to the thermal length scale, i.e., such as $r \leqslant \ell_{T} \sim h / 2$. This leads to

$$
\begin{aligned}
\langle\nabla T\rangle_{z} & =\frac{1}{2 \phi_{\max }} \int_{-\phi_{\max }}^{\phi_{\max }}(\nabla T)_{z} \mathrm{~d} \phi \\
& \simeq \frac{1}{2 \phi_{\max }} \sum_{-\phi_{\max }}^{\phi_{\max }}(\nabla T)_{z} \Delta \phi(z),
\end{aligned}
$$

with $\phi_{\max }=\phi(z, r=h / 2)$ the polar angle corresponding to the largest distance to beam axis to be considered. The discrete summation is used since we numerically computed the temperature field ( $\Delta \phi$ is the corresponding angle step).

The mean value of the temperature gradient, $\langle\nabla T\rangle=\|\langle\nabla T\rangle\|$, is finally given by the average of the mean values calculated in each plane, each being weighted by the number of terms of the sum in eq. 24. Table I summarizes the values obtained for the investigated channel aspect ratio. A weak dependance in beam waist can be observed, which weakens for increasing channels heights as $h / 2$ fixes the thermal characteristic length.

\section{B. Temperature gradient dependance of the thermocapillary velocity}

Having characterized the temperature field produced by optical absorption of a laser beam, we can now represent the experimental data of Fig. 4 versus the temperature gra- 
Table I. Mean temperature gradient per unit of optical power, $\langle\nabla T\rangle_{1} / P$ and $\langle\nabla T\rangle_{2} / P$, for the two considered geometries. Subscript 1 corresponds to a channel of width $w=200 \mu \mathrm{m}$ and height $h=30 \mu \mathrm{m}$; subscript 2 corresponds to $w=100 \mu \mathrm{m}$ and $h=50 \mu \mathrm{m}$.

\begin{tabular}{ccccc}
\hline \hline$\omega_{0}(\mu \mathrm{m})$ & 1.42 & 2.85 & 5.52 & 9.61 \\
\hline$\langle\nabla T\rangle_{1} / P\left(\mathrm{~K} \mathrm{~m}^{-1} \mathrm{~W}^{-1}\right)$ & 3.03 & 2.89 & 2.58 & 2.01 \\
$\langle\nabla T\rangle_{2} / P\left(\mathrm{~K} \mathrm{\mu m}^{-1} \mathrm{~W}^{-1}\right)$ & 6.09 & 5.97 & 5.69 & 5.13 \\
\hline \hline
\end{tabular}

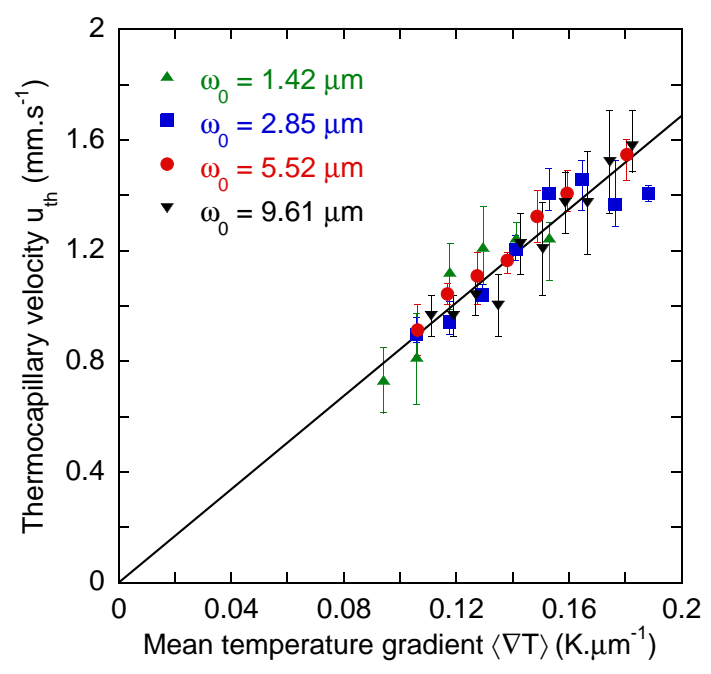

Figure 8. Data of Fig. 4, rescaled in terms of mean temperature gradient on the interface.

dient instead of beam power (Fig. 8). All data points fall onto a single master curve, illustrating a linear behavior. The thermocapillary velocity is thus proportional to the mean temperature gradient. This result could seem expectable as it was already demonstrated when the temperature gradient length scale is large compared to the drop size. However, the major difference here is the fact that laser heating occurs on a length scale that can be much smaller than the drop. We can therefore conclude that, whatever the locality or the non-locality of the heating, the resulting thermocapillary stresses are driven by the mean temperature gradient felt by the droplet interface.

\section{Geometrical dependance of the thermocapillary velocity}

Previous studies [9-13] have considered thermocapillary migration in confined geometries. One the one hand, for bubbles squeezed between two parallel plates, Bratukhin et al. $[9,10]$ 
showed that the thermocapillary velocity remains proportional to the bubble radius, as in the unconfined case. On the other hand, Mazouchi and Homsy [12] and Lajeunesse and Homsy [13] demonstrated that, for elongated bubbles (i.e., confined both horizontally and vertically), the thermocapillary migration mainly results from the flow of the external fluid through the channel corners. In this case, the thermocapillary velocity depends on the smallest dimension of the channel, here its height $h$, but not on the bubble size. In our microchannel, the confinement is also two dimensional. However, we have just demonstrated that the value of a laser-induced temperature gradient is only significant at small polar angles [typically for $\phi<20^{\circ}$, see Fig. 7 (b)], so the thermally-induced external flow through the corners can be neglected. The thermocapillary migration should thus be mainly driven by the flow near the droplet front. To check this hypothesis, we measured the thermocapillary velocity of droplets in microchannels of different aspect ratios.

These experiments were performed with water solution containing E122 food dye. Two reasons motivated this dye choice: (i) the demonstration of the versatility of the method by the use of a food dye, and (ii) a significant reduction of the fluorescence compared to uranine (note that Rhodamine 6G also works but is recognized as a mutagen molecule). The purchased solution was diluted in the water phase at a concentration of $5.5 \%$ in weight. Experiments were performed for three different beam waists, in a range of beam powers comprised between 70 and $230 \mathrm{~mW}$. The lower and upper bounds, which depend on the waist considered, correspond to the blocking and boiling thresholds, respectively.

Since we do not know the absorption of the solution, the measurements performed with E122 are plotted against the mean temperature gradient per unit optical absorption, as represented in the inset of Fig. 9. We can see that the temperature gradient required to reach a given thermocapillary velocity in the $(100 \times 50)$ channel is twice that of the $(200 \times 30)$ one. Therefore, the thermocapillary velocity should be proportional to $w\langle\nabla T\rangle$, as plotted in Fig. 9. As the radius of curvature of the meniscus in the plane of observation is imposed by the channel width, $R=w / 2$, this scaling suggests that the thermocapillary velocity is proportional to the droplet radius. Once again, the behavior expected in unconfined environment is retrieved. This robustness was already demonstrated by Bratukhin et al. $[9,10]$ for squeezed drops in Hele-Shaw cells and we show here that it is still valid in the presence of lateral walls for a local laser heating. The role of the confinement being to reduce the thermocapillary velocity amplitude due to wall friction $[9,10]$, it is not surprising that 


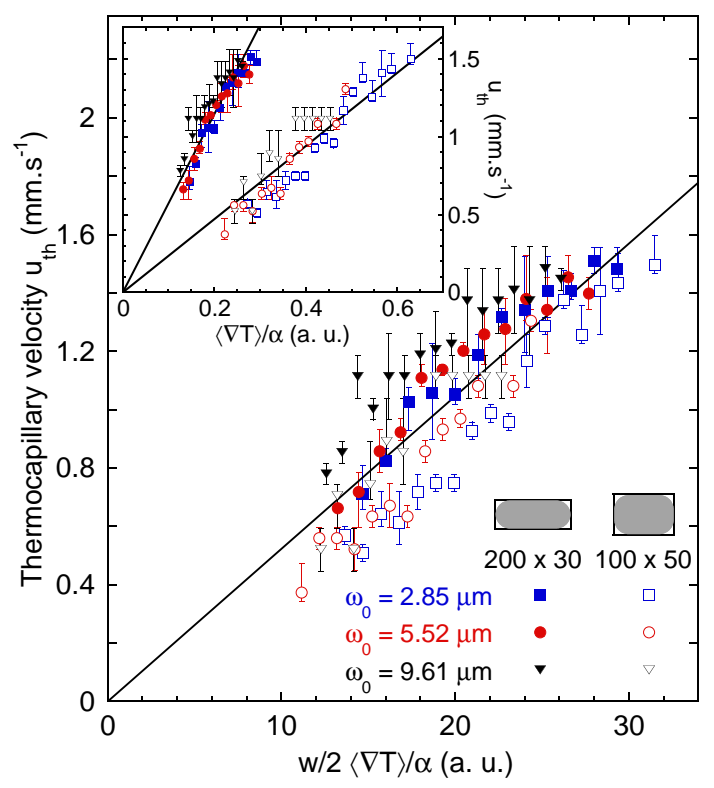

Figure 9. Thermocapillary velocity of E122 food dye droplets in two microchannels, for three distinct beam waists, plotted against the channel half width times the mean temperature gradient per unit optical absorbtion. Inset: same data plotted against the mean temperature gradient per unit optical absorbtion only.

the drop radius dependance is preserved with addition of lateral walls. More surprising is the robustness of this dependance in presence of a local laser heating of length scale smaller than the drop size. This suggests that the important feature is not the droplet size itself but the radius of curvature that drives the thermocapillary migration, as all mechanical effects take place in the vicinity of the beam axis.

Finally, the present results agree well with previous investigations of droplet switching in enlarged (Hele-Shaw-like) channels [26]. Using the uranine solutions we previously found $u_{\mathrm{th}} /(R P) \simeq 0.11 \mathrm{~mJ}^{-1}$, with a beam waist close to $10 \mu \mathrm{m}$ and a $30-\mu \mathrm{m}$-high channel. This value can be compared to the slope of the plot in Fig. 4 obtained at an identical beam waist. Taking $R \sim w / 2=100 \mu \mathrm{m}$, we get $u_{\mathrm{th}} /(R P) \simeq 0.16 \mathrm{~mJ}^{-1}$.

\section{APPLICATION TO OPTOFLUIDIC DROP DISPENSING}

A direct application of the thermocapillary actuation is the interface blocking phenomenon. As shown in Figs. 2 and 3, the motion of the droplet front is interrupted during a defined interval of time, which we call blocking time $\tau_{b}$. During the blocking process, all 


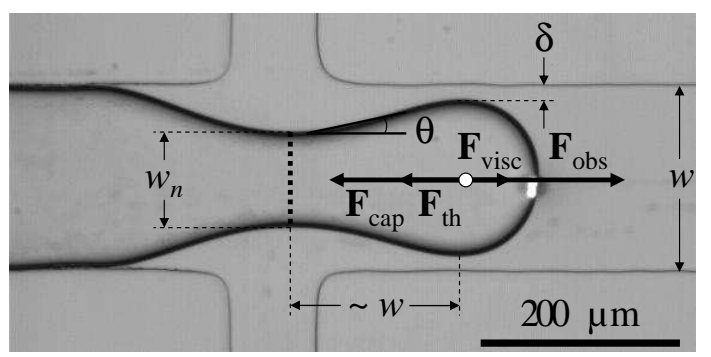

Figure 10. Representation of the main forces acting on a droplet during the blocking process. Channel width and height are $200 \mu \mathrm{m}$ and $30 \mu \mathrm{m}$, respectively.

forces applying on the meniscus equilibrate. These forces may be time-dependant due to the presence of various flows. Therefore, we understand the blocking time as the time at which the front meniscus loses its rest position imposed by the laser beam. In this section, we thus propose a possible mechanism, based on this time-dependant mechanical equilibrium, to describe the experimentally-measured blocking time. The present section is organized as follows. First, we define the system, and the externally applied forces. Their temporal dependance is also estimated from experimental observations, distinguishing when required the pressure-driven and flow-rate-driven cases. The time-dependant equilibrium of forces leads to a relationship between the blocking time and the temperature gradient, which will be compared to measurements. Finally, we show that the opto-capillary blocking is an appealing way of controlling either the droplet volume or the flow rate.

\section{A. Forces involved in the blocking process}

We consider a droplet, blocked during its formation process. As represented in Fig. 10, a neck forms upstream from the beam axis, which eventually (after the end of the blocking process) breaks up. Therefore, this neck represents a relevant limit for the volume of fluid that will further constitute a droplet. This is the reason why hereafter we abusively call "droplet" this volume of fluid. During the blocking process, the oil phase continues to flow around the droplet, through two lateral interstices of width $\delta$. We neglect the top and bottom very thin lubrication films.

The main external forces acting on the droplet are (i) the thermocapillary force, $F_{\mathrm{th}}$, (ii) the viscous force, $F_{\text {visc }}$, resulting from the shear of the interface by the external phase, (iii) the force $F_{\text {obs }}$ resulting from the increase of hydrodynamic resistance due to the obstruction 
of the channel, and (iv) the capillary force, $F_{\text {cap }}$, associated with the difference between upstream and downstream capillary pressure.

\section{Thermocapillary force}

The thermocapillary force is driven by laser-induced Marangoni flows, which is at the origin of the thermocapillary migration. As seen in Ref. [28], and quantitatively demonstrated in the previous section, this force, oriented upstream, is proportional to the apparent interfacial tension gradient. We can thus write the thermocapillary force as

$$
F_{\text {th }} \propto-\left(\frac{\partial \sigma}{\partial T}\right)_{\text {eff }}\langle\nabla T\rangle
$$

with $\langle\nabla T\rangle$ the mean temperature gradient calculated in the previous section. We should look at any possible time-dependance of this force. As previously stated, the temperature distribution is stationary during the blocking process, so the mean temperature gradient is constant. Any temporal variation should thus result from the term $(\partial \sigma / \partial T)_{\mathrm{eff}}$.

According to Khattari et al. [18], for an interface covered with an insoluble surfactant at interfacial concentration $\Gamma$, the effective variation of interfacial tension with temperature expresses as

$$
\left(\frac{\partial \sigma}{\partial T}\right)_{\mathrm{eff}}=\left(\frac{\partial \sigma}{\partial T}\right)_{\Gamma}+\frac{\left(\frac{\partial \sigma}{\partial \Gamma}\right)_{T}}{\left(\frac{\partial^{2} \sigma}{\partial \Gamma^{2}}\right)_{T}}\left[\frac{1}{T}\left(\frac{\partial \sigma}{\partial \Gamma}\right)_{T}-\frac{\partial^{2} \sigma}{\partial T \partial \Gamma}\right] .
$$

The relationship between interfacial tension (or surface pressure, $\Pi=\sigma_{0}-\sigma$, with $\sigma_{0}$ the interfacial tension of a clean interface) and interfacial concentration is usually given at equilibrium by a surface equation of state, such as Langmuir, Frumkin, or van der Waals equations $[37,38]$. However, these commonly-used equations of state apply for dilute solutions and therefore cannot be invoked for describing an interface well above the CMC. On the other hand, Wang et al. [39] measured the surface pressure of a monolayer of Span 80 covering a free surface of phosphate-buffered saline solution $\left(\sigma_{0}=73 \mathrm{mN} \mathrm{m}^{-1}\right)$, in which the surfactant is insoluble. The experimental plot (Ref. [39], Fig. 5) exhibits an inflexion point at a molecular area of $44 \AA^{2}$ per molecule (corresponding to $\Gamma=3.8 \mu \mathrm{mol} \mathrm{m}^{-2}$ ). From these data, we deduce a $\sigma(\Gamma)$ plot, represented in Fig. 11. At high interfacial concentrations, i.e. above $4 \mu \mathrm{mol} \mathrm{m}{ }^{-2}$, the surface tension decays to an asymptotic value $\sigma_{\infty}$, close to $25 \mathrm{mN} \mathrm{m}^{-1}$, definitely ruling out the usual equations of state which all diverge. Since, 


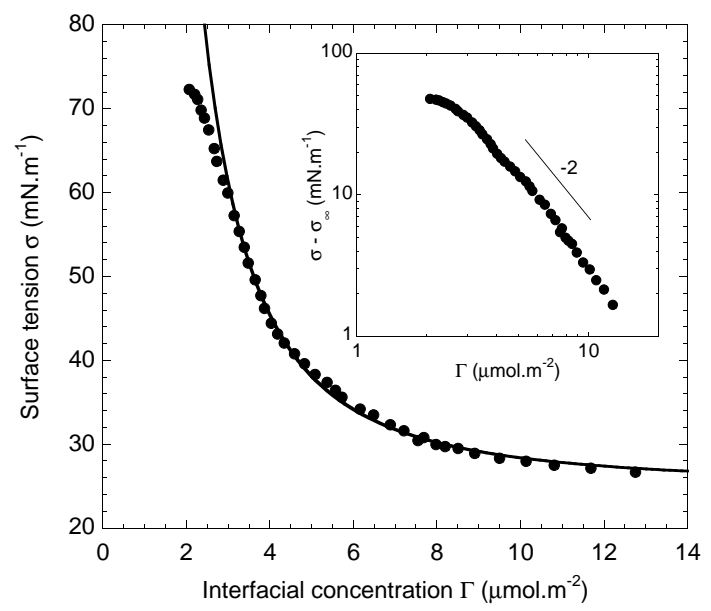

Figure 11. Variation of surface tension at air-water interface with surface concentration of Span 80, deduced from the isotherm measured at $23^{\circ} \mathrm{C}$ by Wang et al. (Figure 5 in Ref. [39]). Solid line, eq. 27, fits the data at high concentrations. Inset: Same data, the surface tension being rescaled by subtracting the asymptotic value $\sigma_{\infty} \simeq 25 \mathrm{mN} \mathrm{m}^{-1}$, plotted in logarithmic scale.

to the best of our knowledge, no theoretical background exist for describing high interfacial concentrations, we look for a simple empirical law. As suggested by the inset of Fig. 11, the actual surface tension corrected by its asymptotic value can reasonably be fitted by a simple $\Gamma^{-2}$ power law, yielding

$$
\sigma=\sigma_{\infty}+\frac{\xi}{\Gamma^{2}}
$$

with $\sigma_{\infty} \simeq 25 \mathrm{mN} \mathrm{m}^{-1}$ the equilibrium surface tension at CMC.

From eqs. 26 and 27, we get

$$
\left(\frac{\partial \sigma}{\partial T}\right)_{\mathrm{eff}}=\frac{\partial \sigma_{\infty}}{\partial T}+\frac{1}{\Gamma^{2}}\left(\frac{2}{3} \frac{\xi}{T}+\frac{1}{3} \frac{\partial \xi}{\partial T}+2 \frac{\xi}{\Gamma} \frac{\partial \Gamma}{\partial T}\right),
$$

which can be positive, providing that the $\Gamma^{-2}$ term is positive and greater than $-\partial \sigma_{\infty} / \partial T$. While the above analysis is based on insoluble surfactants, we will extend it to our hexadecane-water system, and assume that the expression 28 remains qualitatively valid. We can thus expect that the thermocapillary force depends strongly on the interfacial concentration.

In a previous work [26], we estimated $(\partial \sigma / \partial T)_{\text {eff }}$ to be in the $\mathrm{mN} \mathrm{m}^{-1} \mathrm{~K}^{-1}$ range. On the other hand, the value of $\partial \sigma_{\infty} / \partial T \simeq-0.055 \mathrm{mN} \mathrm{m}^{-1} \mathrm{~K}^{-1}$ measured in Ref. [27] means that this term can be neglected in eq. 28. Furthermore, the variation of temperature in our case is relatively small: the maximum temperature rise expected along the beam axis represents 
about $25 \%$ of the ambiant temperature. As a result, we consider that the thermocapillary force approximately scales as $\Gamma^{-2}$, independently from the temperature rise.

Is the surfactant coverage likely to vary during the blocking? Even though we are not able to measure directly the concentration, several remarkable features can be observed, which lead us to think it does. Figure 12 is a series of frames representing the oil phase, downstream from the laser beam, during the blocking process. We especially focus on the channel centerline. Immediately after the blocking starts, we observe a thin trail downstream from the laser beam (underlined by the dashed line on the second frame), composed of unresolved objects advected by the oil flow. This trail lasts during the whole blocking, suggesting that it is composed of excess surfactant molecules which are ejected from the droplet front as the Marangoni flow continuously brings additional molecules. After about $5 \mathrm{~s}$, several dark objects (indicated by the arrows in the third frame) are also emitted and flow downstream along the channel centerline. We suppose that these objects are microdroplets, ejected as the interface is locally saturated by the surfactant. Progressively, more and more objects are formed: as can be seen on the two last frames, they accumulate at the stagnant point near the beam, forming a tip, and then eject on the channel centerline.

The observation of discrete microdroplets after several seconds suggests that the surfactant interfacial concentration increases and reaches a threshold packing value above which the interface folding and microdroplet ejection occurs. This increase in concentration results from the balance between (i) the observed flux of ejection (desorbing flux), and (ii) a fast adsorption on the highly strained interface. However, the physical mechanisms driving these fluxes, and therefore the temporal evolution of the surfactant coverage, are not identified. We thus need a simple expression describing a saturated increase in interfacial concentration. We choose

$$
\Gamma(t)=\Gamma_{\infty}-\Gamma_{0} \mathrm{e}^{-t / \tau_{s}}
$$

where $\Gamma_{\infty}$ and $\left(\Gamma_{\infty}-\Gamma_{0}\right)$ are the largest-packing and initial interfacial concentrations, respectively. The characteristic time $\tau_{s}$ would reasonably be the time at which the first microdroplets appear, i.e. $\tau_{s} \sim 5 \mathrm{~s}$ for the experimental conditions of Fig. 12.

Considering the above expression, the time-dependant thermocapillary force could finally be written as

$$
F_{\mathrm{th}}(t) \simeq-\frac{\beta}{\left(\Gamma_{\infty}-\Gamma_{0} \mathrm{e}^{-t / \tau_{s}}\right)^{2}}\langle\nabla T\rangle
$$




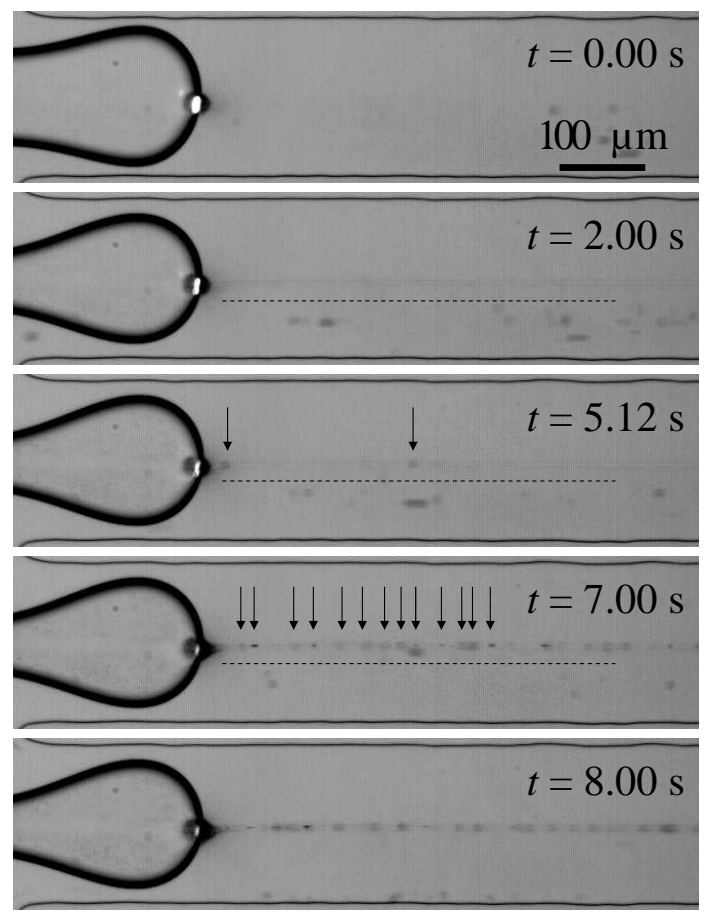

Figure 12. Visualization of the ejection of excess surfactant during the blocking process. Pressuredriven case $\left(\Delta p_{o}=3928 \mathrm{~Pa}\right.$ and $\left.\Delta p_{w}=3897 \mathrm{~Pa}\right), P=127 \mathrm{~mW}$, and $\omega_{0}=2.58 \mu \mathrm{m}$.

with $\beta$ a constant of proportionality.

\section{Viscous force}

The shear stress exerted by the oil phase on a surface $S$ by a velocity gradient $U_{o} / \ell$ is given by $F_{\text {visc }} \sim \eta_{o} U_{o} S / \ell$. Here we consider the force exerted on the rear face of the droplet, which makes a mean angle $\theta$ with the direction of the main flow (see Fig. 10). This force applies on a surface $S \simeq 2 h w \sin \theta$ (the factor 2 accounting for the two sides). The characteristic length defining the velocity gradient is typically the orifice width $\delta$, and the characteristic velocity is given by the flow rate through the section $2 h \delta$ of the interstice, $Q_{\text {int }}$, divided by this section. Therefore we get

$$
F_{\text {visc }} \sim \eta_{o} \frac{w}{\delta^{2}} Q_{\text {int }} \sin \theta
$$

as previously reported by Garstecki et al. [40]. 


\section{Obstruction force}

When a droplet partly fills the microchannel, it increases its hydrodynamic resistance. For a rectangular interstice of section $h \delta$, with $\delta \ll h$, through which oil flows at a rate $Q_{\text {int }} / 2$, the pressure drop over the droplet length $L$ is given by [41]

$$
\Delta p \simeq 12 \eta_{o} \frac{L}{h \delta^{3}} \frac{Q_{\text {int }}}{2},
$$

where the pressure-to-(mass)-flow rate ratio defines the hydrodynamic resistance. Our geometry consists of two parallel obstructions, whose length is of the order of $w$. The force exerting on the surface $S \simeq 2 h w \sin \theta$ is therefore

$$
F_{\text {obs }} \sim 12 \eta_{o} \frac{w^{2}}{\delta^{3}} Q_{\text {int }} \sin \theta
$$

The pressure-driven and flow-rate-driven cases should be distinguished here. If the flow is imposed at constant pressure, an increase in hydrodynamic resistance will result in a decrease in flow rate, keeping the obstruction force constant. Conversely, when the oil flow rate is imposed, this force should vary with the hydrodynamic resistance. In this last case we get $Q_{\text {int }}=Q_{o}$.

This force plays a major role in confined geometries due to its $\delta^{-3}$ scaling. It has previously been invoked for explaining droplet formation in such configurations [40, 42]. We note indeed that this force is larger, by at least one order of magnitude, than the viscous force, and the ratio diverges as $\delta$ vanishes. Therefore, we will neglect the viscous force in the following.

\section{Capillary force}

The Laplace pressure jump across the interface $\Delta p_{L}$ is given by $\Delta p_{L} \simeq \sigma\left(R_{\|}^{-1}+R_{\perp}^{-1}\right)$, $R_{\|}$and $R_{\perp}$ being the in-plane and out-of-plane algebraic radii of curvature, respectively. At the front of the droplet, the interface exerts on the water phase the pressure $\Delta p_{L}^{\text {down }} \simeq$ $-\sigma(2 / h+2 / w)$. At the neck, the Laplace pressure jump between oil and water is given by $\Delta p_{L}^{\text {up }} \simeq-\sigma(2 / h-1 / w)$. The total pressure difference across the droplet is therefore

$$
\Delta p_{L}=\Delta p_{L}^{\text {down }}-\Delta p_{L}^{\text {up }} \simeq-3 \frac{\sigma}{w}
$$



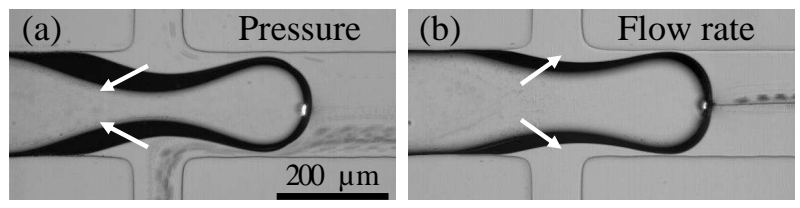

Figure 13. Overlay of successive frames illustrating the evolution of the droplet shape during the blocking process. The white arrows indicate the direction of the temporal evolution. Flow is imposed at constant (a) pressure $\left(\Delta p_{o}=3928 \mathrm{~Pa}\right.$ and $\left.\Delta p_{w}=3897 \mathrm{~Pa}\right)$ or (b) rate $\left(Q_{o}=\right.$ $0.6 \mu \mathrm{L} \mathrm{min}^{-1}$ and $\left.Q_{w}=0.12 \mu \mathrm{L} \mathrm{min}^{-1}\right)$; in both case $P=87 \mathrm{~mW}$, and $\omega_{0}=2.58 \mu \mathrm{m}$.

the minus sign indicates that this stress is oriented upstream. The corresponding force is obtained by multiplying this pressure by the droplet cross-sectional area at the neck, $w_{n} h$,

$$
F_{\text {cap }} \simeq-3 \sigma \frac{h}{w} w_{n}
$$

with $w_{n}$ the neck width. This force opposes to the increase in interfacial area, and therefore has a stabilizing effect.

We finally get a simplified balance composed of three forces: the capillary and thermocapillary forces, which retain the droplet, and the obstruction force, which promotes release. Let us turn to their temporal evolution.

\section{B. Temporal evolution of the droplet shape during blocking}

As seen above, the thermocapillary force is assumed to vary with surfactant coverage during the blocking process. The two other forces strongly depend on the droplet shape. Figure 13 represents the temporal evolution of the interface during the blocking process. At imposed pressure [Fig. 13 (a)], the neck globally thins, whereas it grows at imposed flow rate [Fig. 13 (b)]. We shall thus consider separately the pressure and flow rate cases.

\section{Pressure-imposed flow}

When the flow is imposed at constant pressure, the obstruction force is assumed to be constant, as discussed above. On the other hand, the temporal evolution of the capillary force can be deduced from the thinning dynamics of the neck, which is presented in Fig. 14 for four different droplets at fixed pressure drop values at the microchannel inlets $\left(\Delta p_{o}=3928 \mathrm{~Pa}\right.$ 
and $\Delta p_{w}=3897 \mathrm{~Pa}$ ). The thinning, initially fast, slows down after about $1 \mathrm{~s}$, and saturates at longer times (after $4 \mathrm{~s}$ ). This dynamics does not depend on the beam power. As pointed out in the inset of Fig. 14, such an evolution can be described, from an empirical point of view, by an exponential law like

$$
w_{n}(t)=w_{n}^{0} \mathrm{e}^{-t / \tau_{n}}+w_{n}^{\infty},
$$

with $w_{n}^{0}+w_{n}^{\infty}=w_{n}(t=0)=110 \mu \mathrm{m}, w_{n}^{\infty}=63 \mu \mathrm{m}$, and $\tau_{n} \simeq 0.8 \mathrm{~s}$. Therefore, we can write the time-dependant capillary force as

$$
F_{\text {cap }}(t) \simeq-3 \frac{h}{w} \sigma\left(w_{n}^{0} \mathrm{e}^{-t / \tau_{n}}+w_{n}^{\infty}\right)
$$

Note that this force no longer varies after $4 \mathrm{~s}$, as the droplet shape steadies. Therefore, beyond this saturation the blocking time should be infinite if the thermocapillary force would not decrease with time, suggesting that a mechanism independent from the droplet shape must also be advanced.

The force balance at $t=\tau_{b}$ leads to a relationship between the blocking time and the mean temperature gradient,

$$
\begin{aligned}
\langle\nabla T\rangle & =\frac{\left(\Gamma_{\infty}-\Gamma_{0} \mathrm{e}^{-\tau_{b} / \tau_{s}}\right)^{2}}{\beta} \\
& \times\left[F_{\text {obs }}-3 \frac{h}{w} \sigma\left(w_{n}^{0} \mathrm{e}^{-\tau_{b} / \tau_{n}}+w_{n}^{\infty}\right)\right],
\end{aligned}
$$

which is compared to experimental data in the next section.

\section{Rate-imposed flow}

When the flow is imposed at constant rate, both capillary and obstruction forces should vary. As observed on Fig. 13 (b), the evolution of the droplet shape is composed of both a growth of the neck and a thinning down of the interstice $\delta$. These dynamics are measured, and represented in Fig. 15 for four different droplets, at a given couple of flow rates $\left(Q_{o}=\right.$ $0.6 \mu \mathrm{L} \mathrm{min}^{-1}$ and $\left.Q_{w}=0.12 \mu \mathrm{L} \mathrm{min}^{-1}\right)$. In spite of strong variations in the measurements, the same trend is shared by all the droplets. These dynamics all evidence a linear growth of the droplet volume, as expected when the water flow is fixed. We thus describe both the 


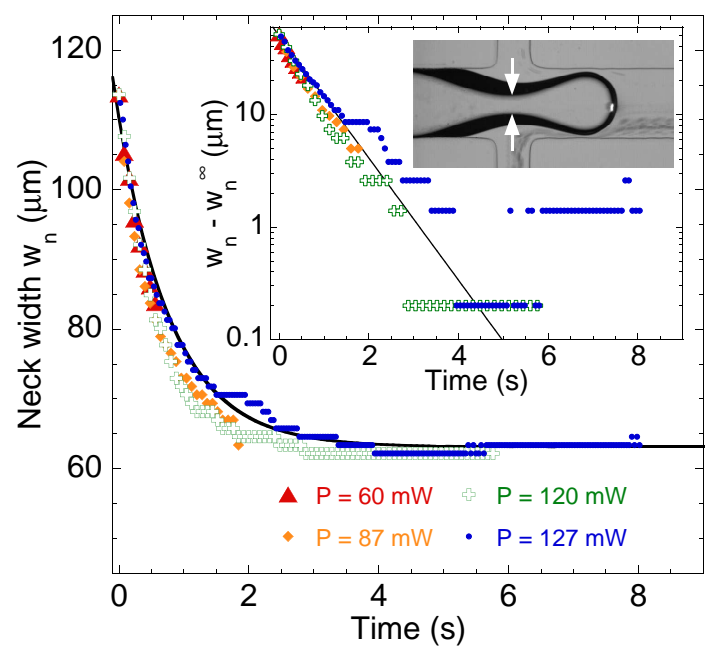

Figure 14. Evolution of the neck width of four blocked droplets, for a pressure-driven flow [same pressures as in Fig. 13 (a)]. The continuous line is a fit with eq. 36. The beam waist is $\omega_{0}=$ $2.58 \mu \mathrm{m}$. Inset: same data, the neck width being corrected by its asymptotic value, in linearlogarithmic scale.

evolution of the neck and interstice width, $w_{n}(t)$ and $\delta(t)$, respectively by

$$
\begin{gathered}
\delta(t)=\delta_{0}-\gamma t \\
w_{n}(t)=w_{n}^{0}+v t,
\end{gathered}
$$

with $\delta_{0} \simeq 10 \mu \mathrm{m}$ and $\gamma$ comprised between 4 and $15 \mu \mathrm{m} \mathrm{s}^{-1}$ for the droplets considered here. Moreover $w_{n}^{0} \simeq 110 \mu \mathrm{m}$ and $v$ is in the $20-50 \mu \mathrm{m} \mathrm{s}^{-1}$ range. Thus we can rewrite the capillary and obstruction forces, respectively, as

$$
\begin{aligned}
& F_{\text {cap }} \simeq-3 \sigma \frac{h}{w}\left(w_{n}^{0}+v t\right) \\
& F_{\text {obs }} \simeq \frac{K}{\left(\delta_{0}-\gamma t\right)^{3}}
\end{aligned}
$$

with $K \sim 12 \eta_{o} w^{2} Q_{o} \sin \theta$. The constant $K$ should not be strictly equal to $12 \eta_{o} w^{2} Q_{o} \sin \theta$ since the latter expression corresponds to a rectangular interstice of homogeneous cross section, which does not match to our experimental situation due to the drop interface curvature. 


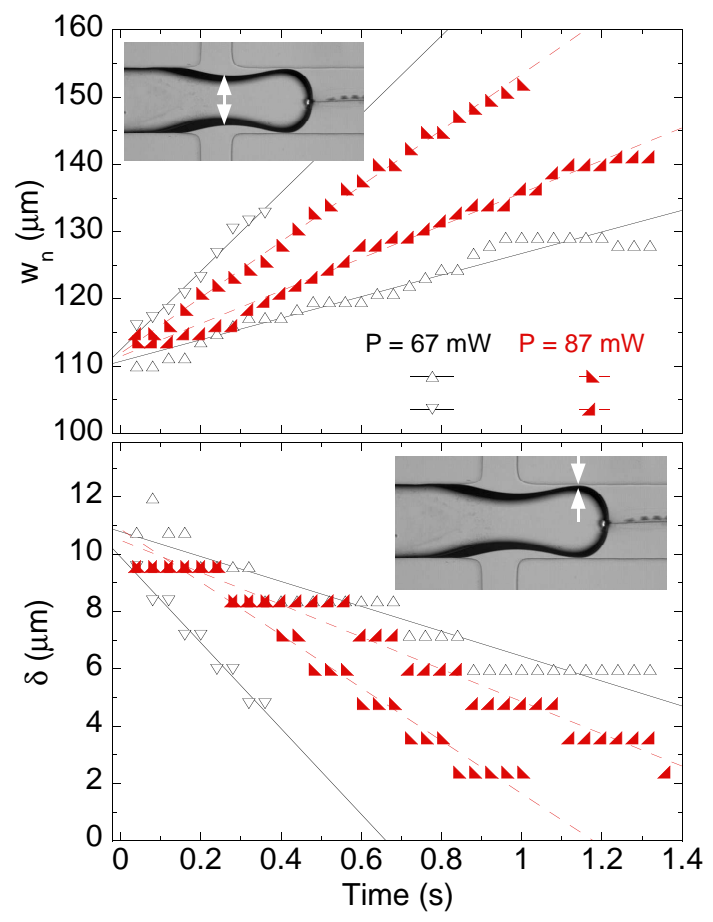

Figure 15. Evolution of the neck width (top) and of the interstice (bottom) of four blocked droplets, for a rate-driven flow [same flow rates as in Fig. 13 (b)]. Beam waist is $\omega_{0}=2.58 \mu \mathrm{m}$.

At $t=\tau_{b}$, the force balance leads to

$$
\begin{aligned}
\langle\nabla T\rangle & =\frac{\left(\Gamma_{\infty}-\Gamma_{0} \mathrm{e}^{-\tau_{b} / \tau_{s}}\right)^{2}}{\beta} \\
& \times\left[\frac{12 \eta_{o} w^{2} Q_{o} \sin \theta}{\left(\delta_{0}-\gamma \tau_{b}\right)^{3}}-3 \sigma \frac{h}{w}\left(w_{n}^{0}+v \tau_{b}\right)\right] .
\end{aligned}
$$

\section{Comparison with experiments}

We measured the blocking time on several successive droplets, produced either by a pressure difference or an imposed flow rate, for a range in beam power comprised between the blocking threshold and the water boiling point, and for three different values of the beam waist. Note that the beam powers involved here are higher than in Sec. III; this can result from the use of uranine coming from another batch, whose optical absorption is lower than the measured value given previously. These data are plotted in Fig. 16.

As represented in the insets of Fig. 16, the blocking time increases with beam power, all the more so the beam waist is small, above a threshold value of the beam power close to $50 \mathrm{~mW}$, for both the pressure [Fig. 16 (a)] and flow rate [Fig. 16 (b)] cases. When 


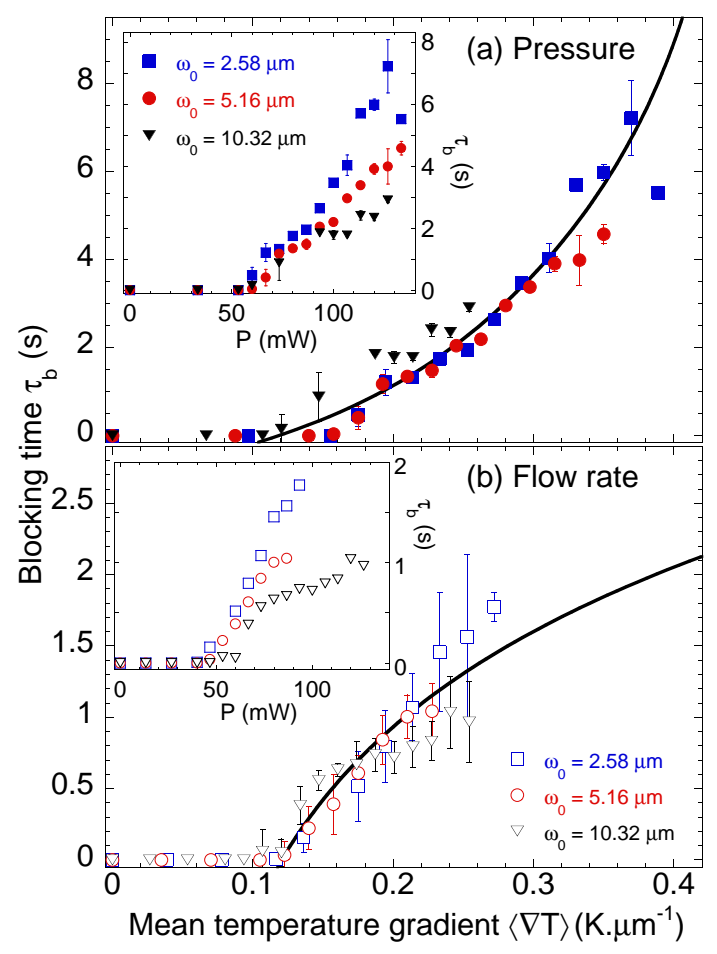

Figure 16. Blocking time, measured for different values of the beam waist in the (a) pressure- and (b) flow-rate-driven cases, plotted against the mean temperature gradient. Solid lines are fits with eqs. 38 (pressure) and 43 (flow rate). Insets: same data, plotted against the beam power. Error bars have been removed on the bottom inset for the sake of clarity.

plotted against the mean temperature gradient, all the data sets fall onto a single behavior, depending only on the fluid injection conditions. As the volume of the drops produced by thermocapillary blocking varies with the incident beam power (see below), this rescaling corroborates the relevance of a local description of opto-capillary actuation. As the produced drops are confined by the channel walls, the curvature of the front interface during the blocking is governed by the channel cross-section independently from the beam power, and then the blocking only depends on the mean temperature gradient.

Experimental data corresponding to the pressure-driven case were fitted by eq. 38 , imposing $F_{\text {obs }}=0.5 \mu \mathrm{N}$, a realistic value according to previous measurements [27]. On the other hand, we fixed $\Gamma_{\infty}=13 \mu \mathrm{mol} \mathrm{m}^{-2}$, which is the maximum value reached by Wang et al. [39] on an air-water interface (see Fig. 11). The characteristic times $\tau_{n}$ and $\tau_{s}$ have been set, from the experimental considerations discussed above, at 0.8 and $5 \mathrm{~s}$, respectively. We considered $\Gamma_{0}$ and $\beta$ as adjustable parameters, and get $\Gamma_{0}=5.15 \mu \mathrm{mol} \mathrm{m}^{-2}$ 
and $\beta=1.32 \times 10^{-16} \mu \mathrm{N} \mathrm{mol}^{2} \mathrm{~m}^{-3} \mathrm{~K}^{-1}$, yielding a thermocapillary force of the order of several $10^{-7} \mathrm{~N}$ in our experimental conditions. On the one hand, the value of $\Gamma_{0}$ means that the initial interfacial concentration of surfactant is about $8 \mu \mathrm{mol} \mathrm{m}^{-2}$. The interfacial concentration therefore increases by about $60 \%$ during the blocking. On the other hand, the initial area per molecule is $21 \AA^{2}$. Peltonen et al. measured a molecular area of $37 \AA^{2}$ for Span 80 at CMC, on unstrained dodecane-water interfaces [43]. This significantly higher value can result from the fact we base our interpretation on measurements performed at the air-water interface. In air, one can reasonably assume that, for steric reasons, surfactant hydrophobic tails can be packed more closely than in alcane. However, these values remain in the same order of magnitude, and eq. 38 therefore describes very well the experiments at the scaling-law level.

Data corresponding to the flow-rate-driven case were then fitted by eq. 43 , with the values of $\Gamma_{0}$ and $\beta$ previously determined. We imposed the geometrical parameters (see Fig. $15)$ in the measured range: $v=40 \mu \mathrm{m} \mathrm{s}^{-1}, w_{n}^{0}=110 \mu \mathrm{m}, \gamma=2 \mu \mathrm{m} \mathrm{s}^{-1}$, and $\delta_{0}=20 \mu \mathrm{m}$. The values of $\gamma$ and $\delta_{0}$ we used are therefore different from those measured, but remain in the same order of magnitude. Indeed, to write the obstruction force we considered the hydrodynamic resistance of an idealized plane-parallel channel of cross-section $h \times \delta$, with $\delta \ll h$. The real corresponding channel has a larger mean cross section, owing to the local curvature of the meniscus close to the lateral wall. Thus, the cross-section of the interstice is not constant. Furthermore, its mean aspect ratio $\delta / h$ is too large to allow the use of the simplified expression 32, which is valid as long as $\delta / h \ll 1$ [41]. Nevertheless, using values of $\gamma$ and $\delta_{0}$ slightly different from those measured does not jeopardize the simplified above analysis.

The mean angle $\theta$, considered as an adjustable parameter, is found to be $14.6^{\circ}$, which is compatible with the experimental observations.

In this section, we proposed a possible mechanism for explaining the opto-capillary blocking of droplets during their formation. This mechanism involves both the droplet shape, through hydrodynamic forces, and the evolution of surfactant coverage, which influences the thermocapillary blocking force. Moreover, our observation of a positive $(\partial \sigma / \partial T)_{\text {eff }}$ may rely on the simple fact that surfactant is present at a concentration well above the CMC, as usually done in digital microfluidics. This behavior should therefore be qualitatively retrieved with any experiment involving a liquid interface submitted to a temperature gradient, with 
(a) Pressure

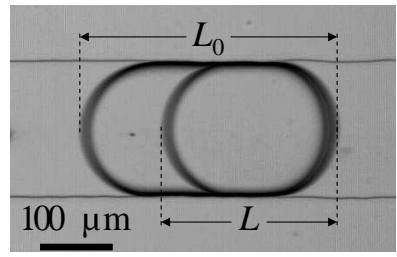

(b) Flow rate

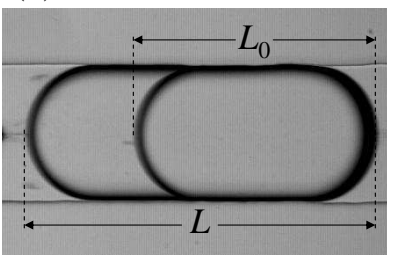

Figure 17. Superposition of two frames comparing blocked (length $L$ ) and unblocked (length $L_{0}$ ) droplets, for the cases where the flow is imposed at constant (a) pressure or (b) rate; in both case $P=87 \mathrm{~mW}$, and $\omega_{0}=2.58 \mu \mathrm{m}$.

a high concentration of surfactant.

\section{Droplet size after release}

As shown above (Figs. 3 and 16), interrupting the imposed flow decreases the frequency of droplet production. Moreover, we observe that the size of the emitted droplets is also modified by the thermocapillary blocking. Figure 17 shows this behavior, by comparing the droplet length in presence $(L)$ and in absence $\left(L_{0}\right)$ of laser, in the pressure [Fig. 17 (a)] and flow rate [Fig. 17 (b)] cases. The experimental conditions of flow allow for the formation of droplets of comparable volume in both case (here $L_{0}=355 \mu \mathrm{m}$ in pressure, $334 \mu \mathrm{m}$ in flow rate). However, it can be noted that the blocking effect results in opposite features: blocked pressure-driven droplets are smaller than the unblocked ones (here $L=240 \mu \mathrm{m}$ ), whereas blocked rate-driven droplets are longer (here $L=480 \mu \mathrm{m}$ ).

We measured the droplet length for the studied range of laser power: these data are represented in Fig. 18. As observed in the inset, below a threshold power of about 50 $\mathrm{mW}$, corresponding to the blocking threshold, the laser heating has no significant effect on the emitted droplets. Above this threshold, shorter droplets are emitted in pressure-driven, and longer in rate-driven flows. Moreover, qualitative behaviors are also different: pressuredriven blocked droplets are about $30 \%$ shorter than those produced without laser, whatever the beam power and waist, while rate-driven blocked droplet size depends on both, growing with power, all the more so the waist is small. This trend should be compared to that observed for the blocking time. At constant flow rate, mass conservation,

$$
L=L_{0}+\frac{Q_{w}}{w h} \tau_{b}
$$




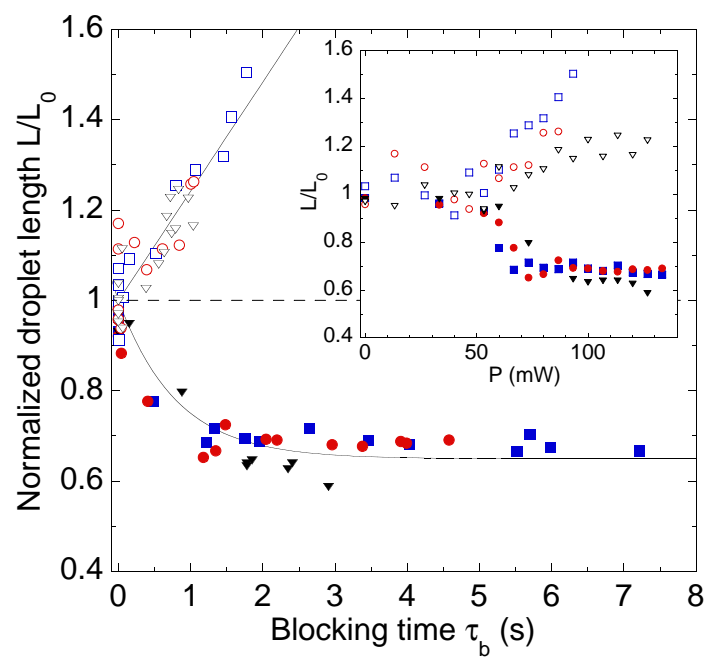

Figure 18. Droplet length normalized by $L_{0}$, represented versus the blocking time, for both pressure-driven (filled symbols) and rate-driven (open symbols) flows. Symbols are the same as in Fig. 16. Continuous lines are fits of the form 36 and 44 ; the dashed line at $L / L_{0}=1$ delimits the pressure and flow-rate data. Inset: same data, plotted versus the beam power.

leads to a linear increase in droplet volume during the blocking time.

The droplet length is thus plotted against the blocking time (Fig. 18). The linear behavior expected for data at constant flow rate (open symbols) is qualitatively retrieved. However, the slope of the straight line is smaller than $Q_{w} /\left(w h L_{0}\right)$, which means that a part of the added volume is excluded from the droplet before its release. Data corresponding to constant pressure experiments (filled symbols) show a fast narrowing of the size at short time droplet blocking (1 s or less), followed by a saturation. This dynamics remind that of the neck width: the decrease in volume can be viewed as resulting from the neck thinning. These data are indeed well fitted by the empiric exponential law used to quantify the neck thinning dynamics (eq. 36).

To conclude this section, we showed that the opto-capillary blocking of drops produced in a confined channel leads to two different behaviors. For a pressure-driven flow, both the volume and the frequency of emission are reduced. The water flow rate is therefore reduced: the device, in this case, behaves as a valve. Conversely, for a rate-driven flow the reduction in frequency of emission is balanced by an increase in droplet volume. As the water flow rate is not modified, this is the droplet size which becomes tunable: such a device can be viewed as a droplet sampler. 


\section{CONCLUDING REMARKS}

We experimentally investigated the laser-induced thermocapillary interaction with a microfluidic droplet generator. This study aims at understanding the specificities linked to strongly localized temperature gradients, and comparing the related behaviors to more classical studies involving large scale uniform temperature gradients to manipulate unconfined droplets. In order to quantitatively investigate the thermocapillary velocity driven by a localized temperature gradient, we considered the case where heating implies an effective increase of the interfacial tension with temperature. Indeed, such a situation is particularly advantageous in the sense that the induced Marangoni streams oppose to the main flow imposed in the microchannel, leading to the possible tuning of the drop velocity down to total blocking, the laser beam behaving as a sort of "soft door" of tunable stiffness. Using this approach, the thermocapillary velocity was characterized as a function of the beam power and waist. Experimental results were then interpreted using the calculated mean temperature gradient imposed by the beam profile and the channel geometry. A single linear master behavior of the measured velocity versus the temperature gradient is raised whatever the beam waist value. The interesting point here is that an analogous behavior is obtained in large-scale temperature gradients while laser-induced temperature gradients are not spatially uniform over the drop size. This strongly suggests that the thermocapillary velocity is in fact proportional to the mean value of the temperature gradient, which obviously coincides to the temperature gradient in the large-scale situation. The channel cross-section dependence was also characterized. Beyond wall friction effects due to the confinement, we showed that the radius of curvature of the front interface also plays a major role, generalizing again the unconfined large-scale gradient situation in which the thermocapillary velocity is known to vary as the droplet radius.

Considering the reverse nature of the optocapillary migration due to $(\partial \sigma / \partial T)_{\text {eff }}>0$, and the possibility to slow down the droplet formation in a microchannel, we then characterized this blocking effect, by measuring the blocking time before droplet release and the corresponding droplet size for several experimental conditions. A simplified force balance gave a possible interpretation, at the scaling-law level, of our measurements. For instance, we noticed a qualitative difference, in the time-dependant forces, between pressure-driven and rate-driven flows, due to a different evolution of the shape of a forming droplet. Moreover, 
the order of magnitude of the physical quantities involved are realistic compared to those previously reported in the literature. A full understanding of the involved processes would nevertheless require a more complete description of the hydrodynamic and physicochemical processes, which to date represents a numerical challenge. This task also depends on probably an even more difficult challenge, a general understanding, beyond our empirical interpretations, of the temperature variation of the interfacial tension in presence of soluble surfactants, including dynamical tension effects as interface blocking occurs in a few milliseconds. Pure thermal variations and Marangoni-driven surfactant concentration variations clearly couple together, and interfacial physical-chemistry studies, including the dynamical behavior of surfactant molecules under strong stresses, are clearly missing.

From an applied point of view, we demonstrated the relevance of this technique for handling droplets flowing at velocities up to the $\mathrm{mm} \mathrm{s}^{-1}$ range, which is comparable to other techniques involving external fields (e.g. electroosmosis [44, 45], or (di)electrophoresis $[46,47])$. More interestingly, the optocapillary interaction with a droplet generator can lead either to a valve, or to a droplet sampler, depending on the fluid injection conditions which are imposed.

\section{ACKNOWLEDGMENTS}

This work was partly supported by the Conseil régional d'Aquitaine through grant No. 20061102030. We gratefully acknowledge Rhodia's Laboratory of Future (LoF) for access to microfabrication facilities, and J.-B. Salmon for help this field. We also thank R. Wunenburger, I. Cantat, and H. Kellay for fruitful discussions, and G. Jonusauskas for help and advice in optical absorption and fluorescence measurements.

\section{Appendix A: Thermal transfer through wetting oil film}

In order to calculate the temperature field due to the laser heating (Sec. IV), we assumed that thermal transfers through the hexadecane wetting films could be neglected. To estimate the error resulting from this simplification, we consider here a thin film of hexadecane, of thickness $\varepsilon$, sandwiched between water and glass layers (see Fig. 19). Since the thickness of this film is very small compared to the channel height, $\varepsilon \ll h$, and even smaller than the 


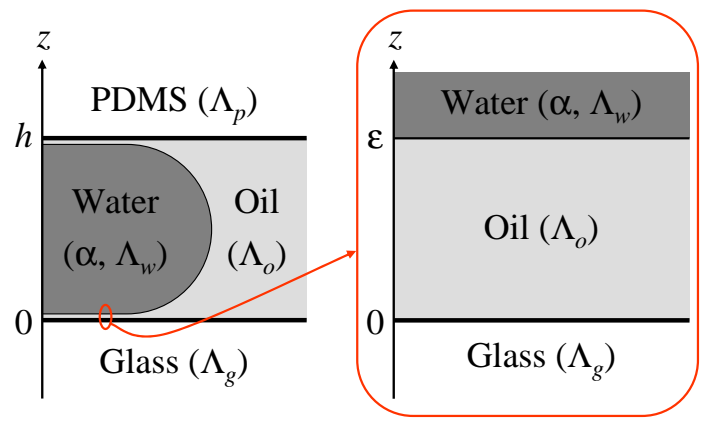

Figure 19. Schematical side view of the meniscus: detail of the wetting oil film.

glass substrate, the two bounding water and glass layers can be considered as semi-infinite. Moreover, as hexadecane and PDMS have similar thermal conductivities, we can also assume the upper film to be thermally matched with the PDMS layer.

As previously detailed (see Sec. IV), we get, in water,

$$
\tilde{T}_{w}(k, z)=C_{w}(k) \mathrm{e}^{-k z}+\frac{\alpha}{\Lambda_{w}} \frac{\tilde{I}(k)}{k^{2}},
$$

with (subscripts $w, o$, and $g$ denote water, oil, and glass, respectively, and $\Lambda_{i j}=\Lambda_{i} / \Lambda_{j}$ )

$$
\begin{aligned}
& C_{w}(k)=\frac{\alpha}{\Lambda_{w}} \frac{\tilde{I}(k)}{k^{2}} \\
& \times \frac{\left(1-\Lambda_{g o}\right) \mathrm{e}^{-2 k \varepsilon}-\left(1+\Lambda_{g o}\right)}{\left(1+\Lambda_{g o}\right)\left(1+\Lambda_{w o}\right)-\left(1-\Lambda_{g o}\right)\left(1-\Lambda_{w o}\right) \mathrm{e}^{-2 k \varepsilon}} .
\end{aligned}
$$

A development at first order in $k \varepsilon$ leads to

$$
\begin{aligned}
C_{w}(k) & =\frac{\tilde{I}(k)}{k^{2}} \frac{2 \alpha \Lambda_{g w}}{\left(1-\Lambda_{g o}\right)\left(1-\Lambda_{w o}\right)-\left(1+\Lambda_{g o}\right)\left(1+\Lambda_{w o}\right)} \\
& \times\left[\frac{1}{\Lambda_{o}}+\left(\frac{1}{\Lambda_{g}}-\frac{1}{\Lambda_{o}}-\frac{\left(1-\Lambda_{g o}\right)\left(1-\Lambda_{w o}\right)}{\Lambda_{g}+\Lambda_{w}}\right) k \varepsilon\right]+\mathcal{O}\left(k^{2} \varepsilon^{2}\right) .
\end{aligned}
$$

Since $\Lambda_{g o} \simeq 10$ and $\Lambda_{w o} \simeq 4$, we can write

$$
\tilde{T}_{w}(k, z) \simeq \frac{\alpha}{\Lambda_{w}} \frac{\tilde{I}(k)}{k^{2}}\left(1-\frac{5}{7} \mathrm{e}^{-k z}+\frac{15}{7} k \varepsilon \mathrm{e}^{-k z}\right) .
$$

The thermal transfer through the wetting film corresponds to the term in $k \varepsilon$ in eq. A4. In the real space, it can be written as

$$
T_{w}^{\varepsilon}(r, z) \simeq \frac{\alpha P}{2 \pi \Lambda_{w}} \int_{0}^{\infty} \frac{15}{7} \varepsilon \mathrm{e}^{-k^{2} \omega_{0}^{2} / 8-k z} J_{0}(k r) \mathrm{d} k,
$$




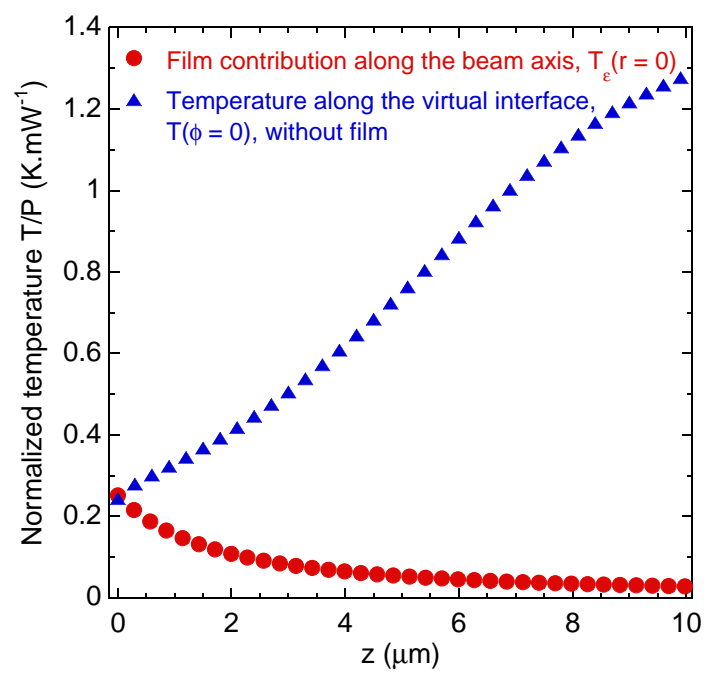

Figure 20. Contribution of the thermal transfer through the wetting film (eq. A6) in the temperature rise along the beam axis in water, compared to the temperature rise calculated without accounting for the films (eq. 20) in a 30- $\mu$ m-high channel. Beam waist is $\omega_{0}=2.85 \mu \mathrm{m}$ in both case.

which reduces, along the beam axis $(r=0)$ and for $z \geqslant 0$, to

$$
T_{w}^{\varepsilon}(0, z) \simeq \frac{15}{4} \sqrt{\frac{2}{\pi}} \frac{\alpha P}{\Lambda_{w}} \frac{\varepsilon}{\omega_{0}} \mathrm{e}^{2 z^{2} / \omega_{0}^{2}} \operatorname{erfc}\left(\sqrt{2} \frac{z}{\omega_{0}}\right)
$$

with $\operatorname{erfc}(x)=2 / \sqrt{\pi} \int_{x}^{\infty} \mathrm{e}^{-t^{2}} \mathrm{~d} t$ the complementary error function. This expression is plotted in Fig. 20 together with the vertical temperature dependance calculated in Sec. IV, for $\omega_{0}=2.85 \mu \mathrm{m}$ and $\varepsilon=\omega_{0} / 10$. Neglecting transfers through the wetting films leads to a significant error in the close vicinity of the boundary, which rapidly decays after a few microns, preventing by the way from a 5 layer-calculation.

\section{Appendix B: Thermal influence of a water-oil interface}

When calculating the temperature field produced by laser heating (Section IV), we have considered a channel filled with water, neglecting the presence of the water-oil interface [see Fig. 5 (b)]. Taking into account the experimental lack of common symmetry between the laser heating and the two-fluid flow separated by the interface [Fig. 5 (a)], and thus the difficulty in getting an analytical expression of the temperature distribution in the present configuration, we estimate the corresponding error using a slightly different solvable, but 
realistic, situation that allows evaluating the influence of the interface on the heat transfer. For the homogeneous situation, we simply consider a water layer of length $\mathrm{L}$ sandwiched by semi-infinite solid layers [Fig. 21 (a)]. The corresponding temperature field is described by eq. 20 and, for the sake of simplicity, we can assume that water is sandwiched left and right by the same material, let us say PDMS which constitute three of the four channel boundaries, since this calculation is supposed to bring insights on the temperature along the flow axis. The length $L$ represents here a distance along the flow direction and then is supposed to be much larger than the channel height $h$, let us say a millimeter, which is large enough compared to the beam waist and keeps the condition $\alpha L<1$; formally, the axial length scale should be more in the centimeter range (the length of the channel), but the presence of side walls in the real situation [Fig. 5 (a)] necessarily imposes a cutoff in the sub-millimeter range. In presence of an interface, we consider instead a water-oil layer in the same geometrical conditions [Fig. 21 (b)], each layer being characterized by a length $L / 2$ (i.e. half a millimeter). The corresponding four-layer situation is almost equivalent to a three-layer case as thermal conductivities of hexadecane and PDMS are very close (see caption of Fig. 5) and hexadecane and PDMS are both transparent at the used optical wavelength. Consequently, both situations can be described by eq. 20, and similarly by eqs. 13 and 17 in oil, the water layer thickness being twice smaller in presence of the waterhexadecane interface. Note finally that the origin of the $x$-axis is different in the two cases of Fig. 21. These choices enable to use directly eqs. 13 and 17 (in oil) and 20 (in water) while keeping the boundaries at $x=0$ and $x=L$, and $x=0$ and $x=L / 2$, respectively.

Moreover, as we a looking for an evaluation of the temperature difference due to the presence of the interface, we compare $T(r=0, x) / P$ for both cases, i.e. along $x$-axis which is here supposed to mimic the flow direction, and at $r=0$ where the temperature variation should be the largest at the interface location. Results are presented in Fig. 22 for a beam waist $\omega_{0}=2.85 \mu \mathrm{m}$ and $L=1 \mathrm{~mm}$; we shifted the $x$-axis in the pure water case [Fig. 21 (a)] to match the position of the center of the water layer with that of the water-oil interface in the other case. Figure 22 shows that the idealized pure water case overestimates the mean temperature by $10 \%$ compared to the real water-oil case. 
(a)

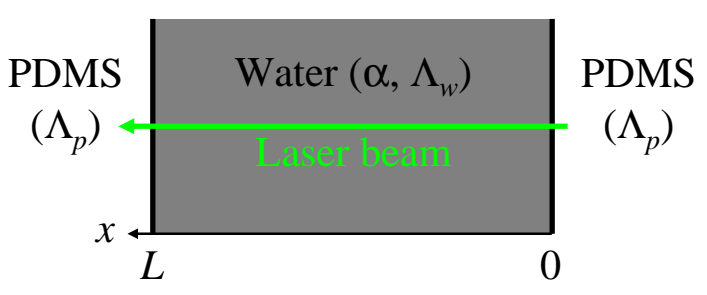

(b)

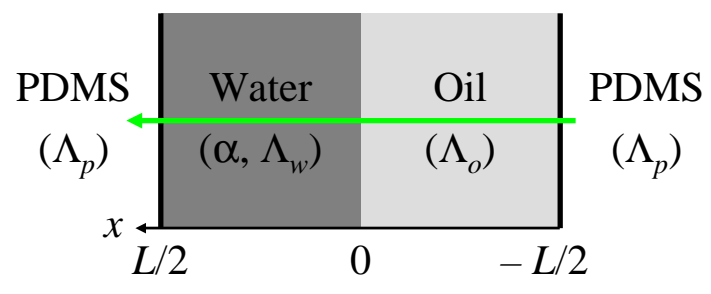

Figure 21. Scheme considered evaluating the difference in laser overheating in absence and presence of a water-oil interface. (a) Channel filled with water. (b) Channel filled with water and oil separated by an interface.

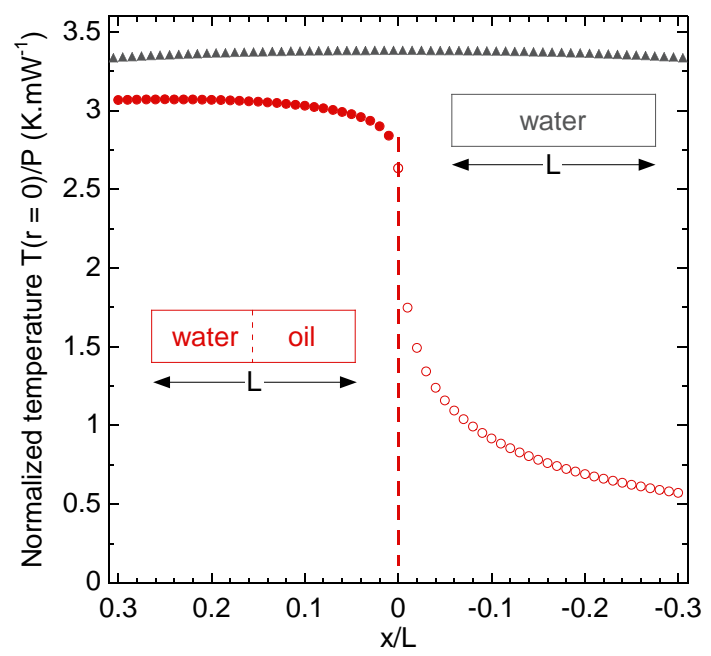

Figure 22. Temperature rise along the channel axis in the absence and the presence of oil. The interface is located at $x=0$. The beam waist is $\omega_{0}=2.85 \mu \mathrm{m}$ and $L=1 \mathrm{~mm}$.

[1] A. Ashkin. Acceleration and trapping of particles by radiation pressure. Phys. Rev. Lett., 24:156-159, 1970.

[2] A. Ashkin, J. M. Dziedzic, J. E. Bjorkholm, and S. Chu. Observation of a single-beam gradient force optical trap for dielectric particles. Opt. Lett., 11:288-290, 1986. 
[3] K. Ladavac and D. G. Grier. Microoptomechanical pumps assembled and driven by holographic optical vortex arrays. Opt. Express, 12:1144-1149, 2004.

[4] J. Leach, H. Mushfique, R. Di Leonardo, M. J. Padgett, and J. M. Cooper. An optically driven pump for microfluidics. Lab Chip, 6:735-739, 2006.

[5] A. Ashkin and J. M. Dziedzic. Radiation pressure on a free liquid surface. Phys. Rev. Lett., 30:139-142, 1973.

[6] A. Casner and J.-P. Delville. Giant deformations of a liquid-liquid interface induced by the optical radiation pressure. Phys. Rev. Lett., 87:054503, 2001.

[7] N. O. Young, J. S. Goldstein, and M. J. Block. The motion of bubbles in a vertical temperature gradient. J. Fluid Mech., 6:350-356, 1959.

[8] M. Hähnel, V. Delitzsch, and H. Eckelmann. The motion of droplets in a vertical temperature gradient. Phys. Fluids A, 1:1460-1466, 1989.

[9] Yu. K. Bratukhin and A. L. Zuev. Thermocapillary drift of an air bubble in a horizontal Hele-Shaw cell. Fluid Dyn., 19:393-398, 1984.

[10] Yu. K. Bratukhin, K. G. Kostarev, A. Viviani, and A. L. Zuev. Experimental study of Marangoni bubble migration in normal gravity. Exp. Fluids, 38:594-605, 2005.

[11] A. Mazouchi and G. M. Homsy. Thermocapillary migration of long bubbles in cylindrical capillary tubes. Phys. Fluids, 12:542-549, 2000.

[12] A. Mazouchi and G. M. Homsy. Thermocapillary migration of long bubbles in polygonal tubes. I. Theory. Phys. Fluids, 13:1594-1600, 2001.

[13] É. Lajeunesse and G. M. Homsy. Thermocapillary migration of long bubbles in polygonal tubes. II. Experiments. Phys. Fluids, 15:308-314, 2003.

[14] K. D. Barton and R. S. Subramanian. The migration of liquid drops in a vertical temperature gradient. J. Colloid Interface Sci., 133:211-222, 1989.

[15] H. S. Kim and R. S. Subramanian. Thermocapillary migration of a droplet with insoluble surfactant: I. Surfactant cap. J. Colloid Interface Sci., 127:417-428, 1989.

[16] H. S. Kim and R. S. Subramanian. Thermocapillary migration of a droplet with insoluble surfactant: II. General case. J. Colloid Interface Sci., 130:112-129, 1989.

[17] Y. S. Chen, Y. L. Lu, Y. M. Yang, and J. R. Maa. Surfactant effects on the motion of a droplet in thermocapillary migration. Int. J. Multiphase Flow, 23:325-335, 1997.

[18] Z. Khattari, P. Steffen, and T. M. Fischer. Migration of a droplet in a liquid: effect of insoluble 
surfactants and thermal gradient. J. Phys.: Condens. Matter, 14:4823-4828, 2002.

[19] A. A. Darhuber, J. P. Valentino, J. M. Davis, S. M. Troian, and S. Wagner. Microfluidic actuation by modulation of surface stresses. Appl. Phys. Lett., 82:657-659, 2003.

[20] R. H. Farahi, A. Passian, T. L. Ferrell, and T. Thundat. Microfluidic manipulation via Marangoni forces. Appl. Phys. Lett., 85:4237-4239, 2004.

[21] B. Selva, V. Miralles, I. Cantat, and M.-C. Jullien. Thermocapillary actuation by optimized resistor pattern: Bubbles and droplets displacing, switching and trapping. Lab Chip, 10:18351840, 2010.

[22] S. Rybalko, N. Magome, and K. Yoshikawa. Forward and backward laser-guided motion of an oil droplet. Phys. Rev. E, 70:046301, 2004.

[23] D. Okawa, S. J. Pastine, A. Zettl, and J. M. J. Fréchet. Surface tension mediated conversion of light to work. J. Am. Chem. Soc., 131:5396-5398, 2009.

[24] C. N. Baroud, J.-P. Delville, F. Gallaire, and R. Wunenburger. Thermocapillary valve for droplet production and sorting. Phys. Rev. E, 75:046302, 2007.

[25] C. N. Baroud, M. Robert de Saint Vincent, and J.-P. Delville. An optical toolbox for total control of droplet microfluidics. Lab Chip, 7:1029-1033, 2007.

[26] M. Robert de Saint Vincent, R. Wunenburger, and J.-P. Delville. Laser switching and sorting for high speed digital microfluidics. Appl. Phys. Lett., 92:154105, 2008.

[27] É. Verneuil, M. L. Cordero, F. Gallaire, and C. N. Baroud. Laser-induced force on a microfluidic drop: origin and magnitude. Langmuir, 25:5127-5134, 2009.

[28] F. Gallaire, C. N. Baroud, and J.-P. Delville. Thermocapillary manipulation of microfluidic droplets: Theory and applications. Int. J. Heat Technology, 26:161-166, 2008.

[29] D. C. Duffy, J. C. McDonald, O. J. A. Schueller, and G. M. Whitesides. Rapid prototyping of microfluidic systems in poly(dimethylsiloxane). Anal. Chem., 70:4974-4984, 1998.

[30] Ordered and disordered patterns in two-phase flows in microchannels.

[31] J. H. Xu, S. W. Li, J. Tan, Y. J. Wang, and G. S. Luo. Controllable preparation of monodisperse $\mathrm{O} / \mathrm{W}$ and $\mathrm{W} / \mathrm{O}$ emulsions in the same microfluidic device. Langmuir, 22:7943-7946, 2006 .

[32] T. Cubaud, M. Tatineni, X. Zhong, and C.-M. Ho. Bubble dispenser in microfluidic devices. Phys. Rev. E, 72:037302, 2005.

[33] A. R. Abate, A. Poitzsch, Y. Hwang, J. Lee, J. Czerwinska, and D. A. Weitz. Impact of inlet 
channel geometry on microfluidic drop formation. Phys. Rev. E, 80:026310, 2009.

[34] A. E. Siegman. Lasers. University Science Books, Stanford, 1986.

[35] M. Lax. Temperature rise induced by a laser beam. J. Appl. Phys., 48:3919-3924, 1977.

[36] M. Abramowitz and I. A. Stegun. Handbook of Mathematical Functions. Dover, New York, 1972.

[37] C.-H. Chang and E. I. Franses. Adsorption dynamics of surfactants at the air/water interface: a critical review of mathematical models, data, and mechanisms. Colloids and Surf. A: Physicochem. Eng. Aspects, 100:1-45, 1995.

[38] J. Eastoe and J. S. Dalton. Dynamic surface tension and adsorption mechanisms of surfactants at the air-water interface. Adv. Colloid Interface Sci., 85:103-144, 2000.

[39] W. Wang, C. C. Moser, and M. A. Wheatley. Langmuir trough study of surfactant mixtures used in the production of a new ultrasound contrast agent consisting of stabilized microbubbles. J. Phys. Chem., 100:13815-13821, 1996.

[40] P. Garstecki, M. J. Fuerstman, H. A. Stone, and G. M. Whitesides. Formation of droplets and bubbles in a microfluidic T-junction-scaling and mechanism of break-up. Lab Chip, 6:437-446, 2006.

[41] H. Bruus. Theoretical Microfluidics. Oxford University Press, New York, 2008.

[42] G. F. Christopher, N. N. Noharuddin, J. A. Taylor, and S. L. Anna. Experimental observations of the squeezing-to-dripping transition in T-shaped microfluidic junctions. Phys. Rev. E, 78:036317, 2008.

[43] L. Peltonen, J. Hirvonen, and J. Yliruusi. The behavior of sorbitan surfactants at the water-oil interface: Straight-chained hydrocarbons from pentane to dodecane as an oil phase. J. Colloid Interface Sci., 240:272 - 276, 2001.

[44] S. Ghosal. Fluid mechanics of electroosmotic flow and its effect on band broadening in capillary electrophoresis. Electrophoresis, 25:214-228, 2004.

[45] A. Ajdari. Electro-osmosis on inhomogeneously charged surfaces. Phys. Rev. Lett., 75:755-758, 1995.

[46] D. R. Link, E. Grasland-Mongrain, A. Duri, F. Sarrazin, Z. Cheng, G. Cristobal, M. Marquez, and D. A. Weitz. Electric control of droplets in microfluidic devices. Angew. Chem. Int. Ed., $45: 2556-2560,2006$.

[47] K. Ahn, C. Kerbage, T. P. Hunt, R. M. Westervelt, D. R. Link, and D. A. Weitz. Dielec- 
trophoretic manipulation of drops for high-speed microfluidic sorting devices. Appl. Phys. Lett., 88:024104, 2006. 